\title{
Efficacy and Safety of Azithromycin for the Treatment of COVID-19: A Systematic Review and Meta-analysis
}

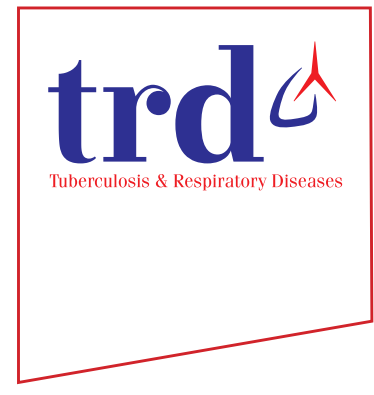

\author{
Ghea Mangkuliguna, B.M. ${ }^{1}{ }^{\mathbb{D}}$, Glenardi, B.M. ${ }^{1}{ }^{\mathbb{C}}$, Natalia, B.M. ${ }^{1}{ }^{\mathbb{D}}$ and Laurentius A. Pramono, M.D., \\ M.Sc. ${ }^{2,3}$ \\ ${ }^{1}$ School of Medicine and Health Sciences, Atma Jaya Catholic University of Indonesia, North Jakarta, ${ }^{2}$ Department of Public \\ Health and Nutrition, School of Medicine and Health Sciences, Atma Jaya Catholic University of Indonesia, North Jakarta, \\ ${ }^{3}$ Department of Internal Medicine, Saint Carolus Hospital, Jakarta, Indonesia
}

Background: The lack of effective medications for coronavirus disease 2019 (COVID-19) has led to a trend of drug repurposing such as the case of azithromycin which shows immunomodulatory and anti-viral effect. Several clinical trials have shown conflicting results. It is currently unclear whether the available evidence is in favor or against the use of azithromycin in COVID-19 patients. Thus, the aim of this study was to investigate the efficacy and safety of azithromycin in COVID-19 patients.

Methods: Four independent reviewers selected relevant studies from PubMed, ScienceDirect, EBSCO, and ProQuest published prior to March 2021. The protocol used in this study has been registered in PROSPERO (CRD42020224967).

Results: We included 17 studies and found that the mortality rate (odds ratio [OR], 0.95; 95\% confidence interval [CI], 0.76-1.19), need of respiratory support (OR, 1.30; 95\% CI, 0.98-1.73), hospitalization rate (standardized mean difference, $0.12 ; 95 \% \mathrm{CI},-0.02$ to 0.27 ), and intensive care unit transfer (OR, 1.21; 95\% CI, 0.79-1.86) of azithromycin-treated group did not differ significantly ( $p>0.05$ ) from those of the control group. Azithromycin treatment did not significantly increase the risk of getting secondary infection (OR, 1.23; 95\% CI, 0.83-1.82), hypoglycemia (OR, 0.73; 95\% CI, 0.38-1.40), gastrointestinal problems (OR, 1.03; 95\% CI, 0.73-1.45) or electrocardiogram abnormalities (OR, 1.16; 95\% CI, 0.94-1.42). The overall quality of evidence ranged from low to very low.

Conclusion: Azithromycin did not result in a superior clinical improvement in COVID-19 patients, although it was welltolerated and safe to use.

Keywords: Azithromycin; COVID-19; Meta-analysis; Systematic Review; Treatment

Address for correspondence: Ghea Mangkuliguna, B.M.

School of Medicine and Health Sciences, Atma Jaya Catholic University of Indonesia, Pluit Raya No. 2, North Jakarta 14440, Indonesia

Phone: 62-21-6693168, Fax: 62-21-6606123

E-mail: mangkuligunaVG1402@yahoo.com

Received: May. 4, 2021

Revised: May. 13, 2021

Accepted: May. 19, 2021

Published online: May. 20, 2021

(c) It is identical to the Creative Commons Attribution Non-Commercial License (http://creativecommons.org/licenses/by-nc/4.0/).

Copyright (C) 2021

\section{Introduction}

Coronavirus disease 2019 (COVID-19) pandemic has infected more than 138 million people with a devastating impact on global health. It has caused more than 2.9 million deaths across 223 countries in the world as of April 16, 2021 . While the majority of people with COVID-19 only develop mild symptoms, about 10\%-15\% people develop severe illness requiring hospitalization and intensive care unit (ICU) admission $^{2}$. There is an immense pressure to find a therapy to improve the prognosis and minimize the mortality rate of COVID-19 patients.

The lack of effective medications for the management of COVID-19 has led to a trend of drug repurposing for an indi- 
cation different from what was initially marketed. One of such cases is the use of macrolide azithromycin, a broad-spectrum antibiotic commonly used to treat respiratory infections ${ }^{3}$, for COVID-19 patients. Besides its bacteriostatic activity, azithromycin has been shown to possess immunomodulatory, antiinflammatory, and anti-viral effect ${ }^{3-5}$. Azithromycin can also lead to a significant improvement of patients with acute respiratory distress syndrome (ARDS) ${ }^{6}$. These findings have served as a rationale for clinical use of azithromycin in COVID-19 treatment, especially for those with moderate-to-severe stage of the disease, although there is a concern on the potential torsadogenic effect of this drug that could lead to cardiac arrest ${ }^{7,8}$. The widespread use of azithromycin in COVID-19 might also be driven by the intention to decrease the risk of bacterial superinfections in patients with a more severe disease ${ }^{9}$. However, several clinical trials have shown conflicting results. Currently it is unclear whether the available evidence is in favor or against the use of azithromycin in COVID-19 patients ${ }^{10-26}$. Existing literature only provided a brief hypothetical explanation on the potential benefit of azithromycin for COVID- $19^{27}$. However, results were not quantitatively measured. Therefore, the objective of this study was to perform a systematic review and meta-analysis of existing clinical studies to further investigate the efficacy and safety of azithromycin in COVID-19 patients.

\section{Materials and Methods}

\section{Study registration and methodology}

This study was reported following the Preferred Reporting Items for Systematic Reviews and Meta-Analyses (PRISMA) criteria $^{28}$. The protocol used in this study had been registered in the International Prospective Register of Systematic Reviews (PROSPERO) (CRD42020224967).

\section{Eligibility criteria}

The following criteria were considered for studies' eligibility: type of study, population, intervention, comparison, and outcome.

\section{1) Type of study}

All types of clinical studies (randomized or non-randomized controlled trials, cohort, case control, cross-sectional) evaluating the role of azithromycin in COVID-19 treatment were included in this study. Reviews, commentaries, conference abstracts, case reports, and case series were excluded.

\section{2) Population}

Patients diagnosed with COVID-19 and admitted to the hospital were included in this study. The severity of COVID-19 ranged from mild to critical conditions based on staging from

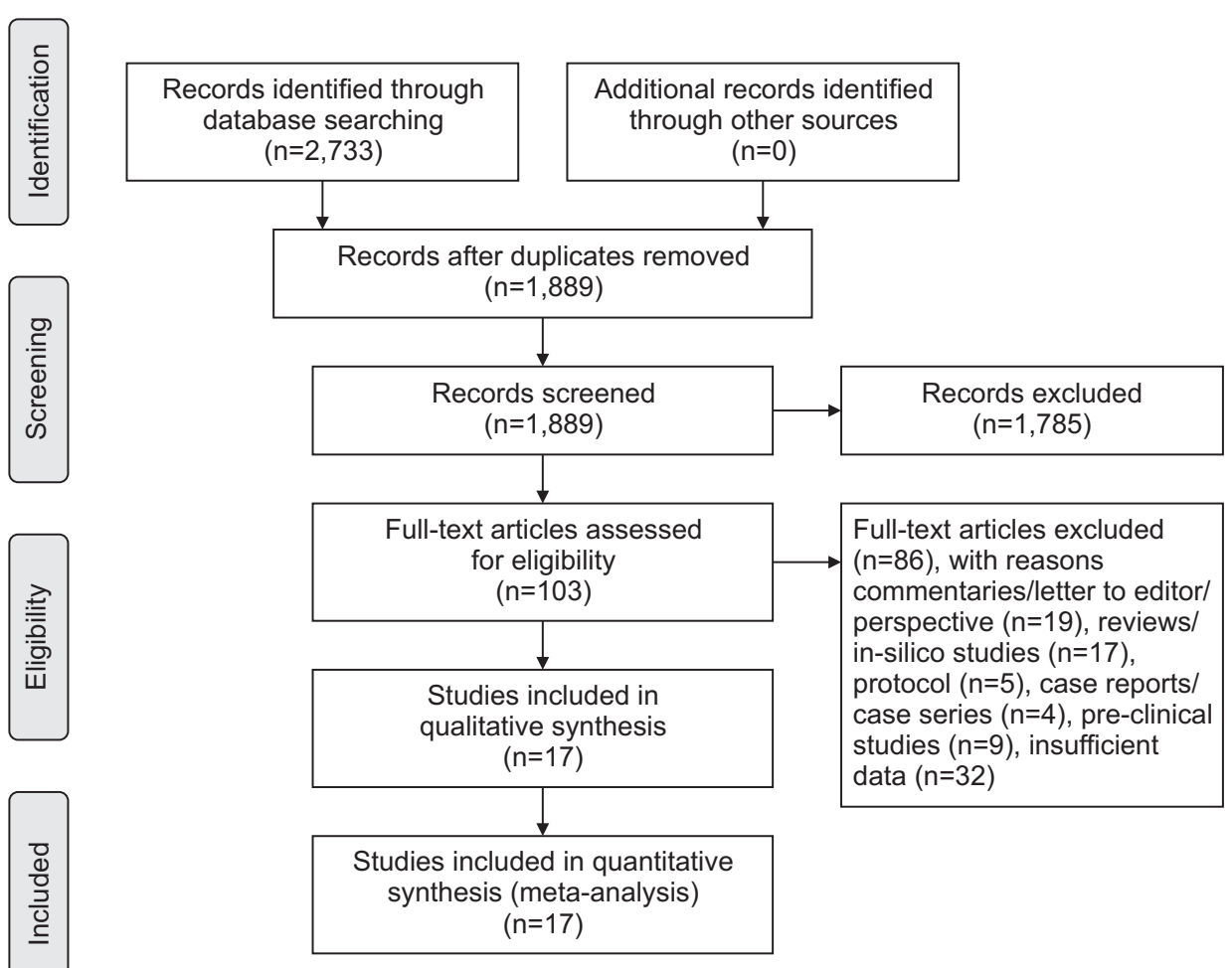

Figure 1. PRISMA Flow Diagram showing the search strategy and the selection process applied to include articles eligible for this meta-analysis. 


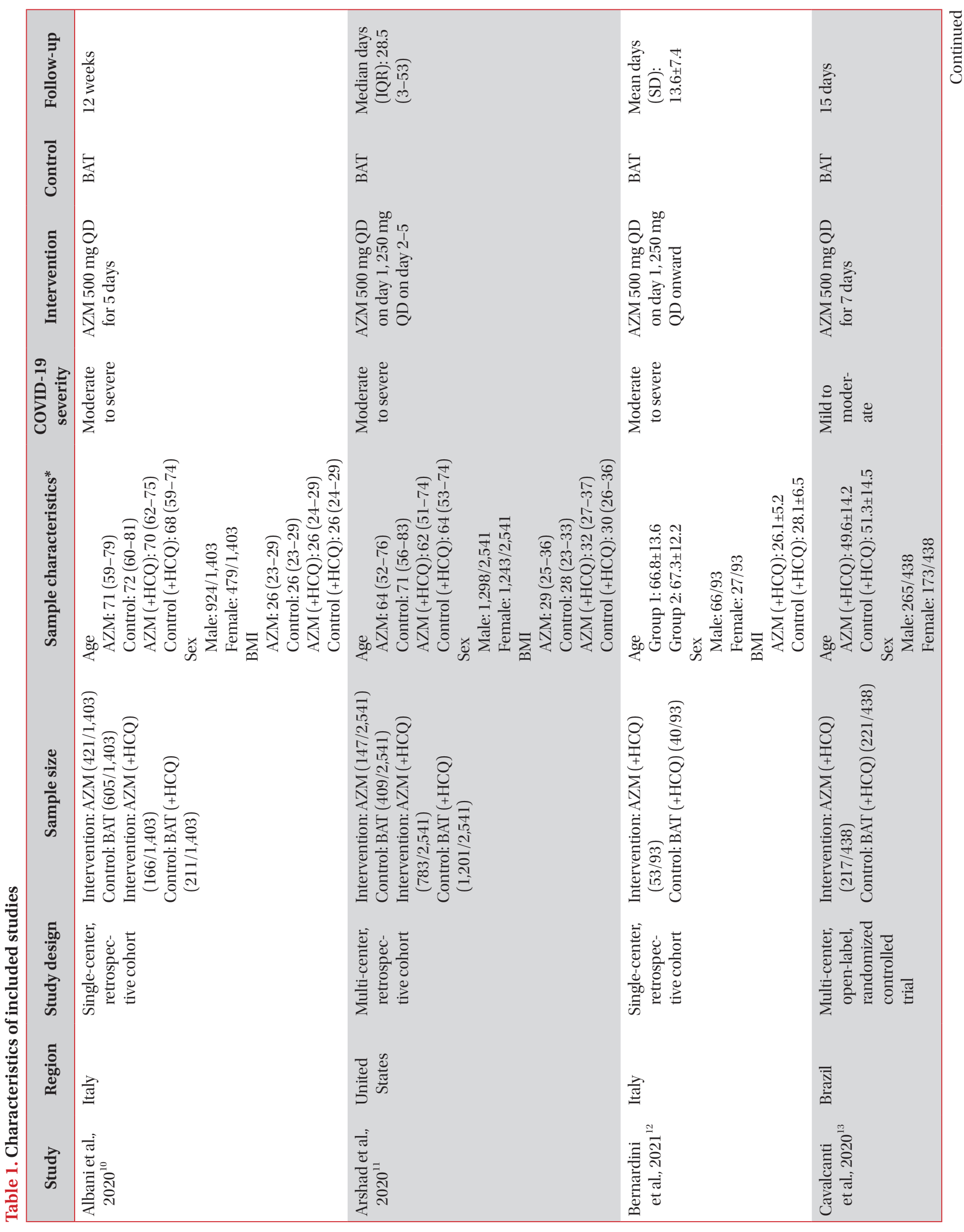




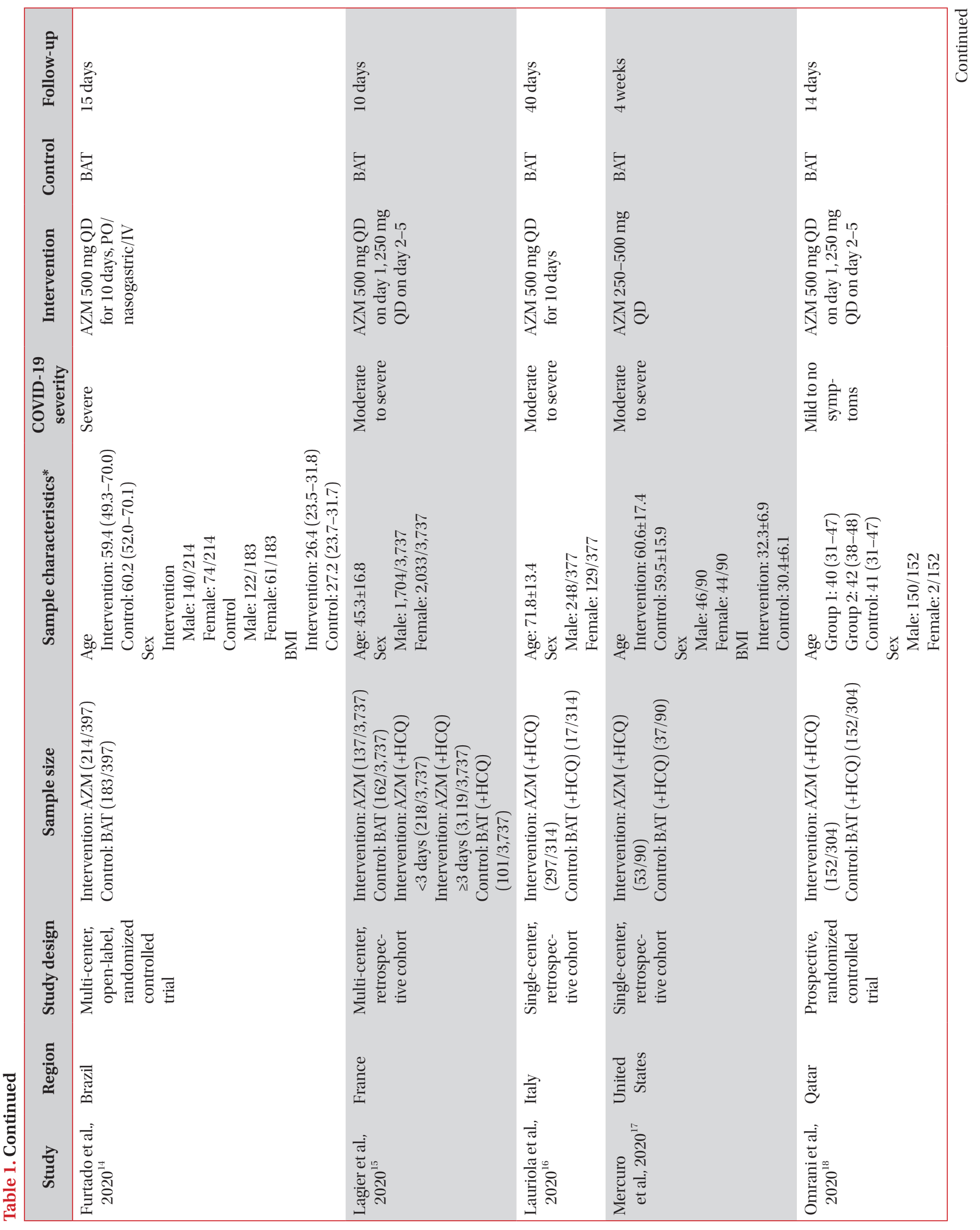




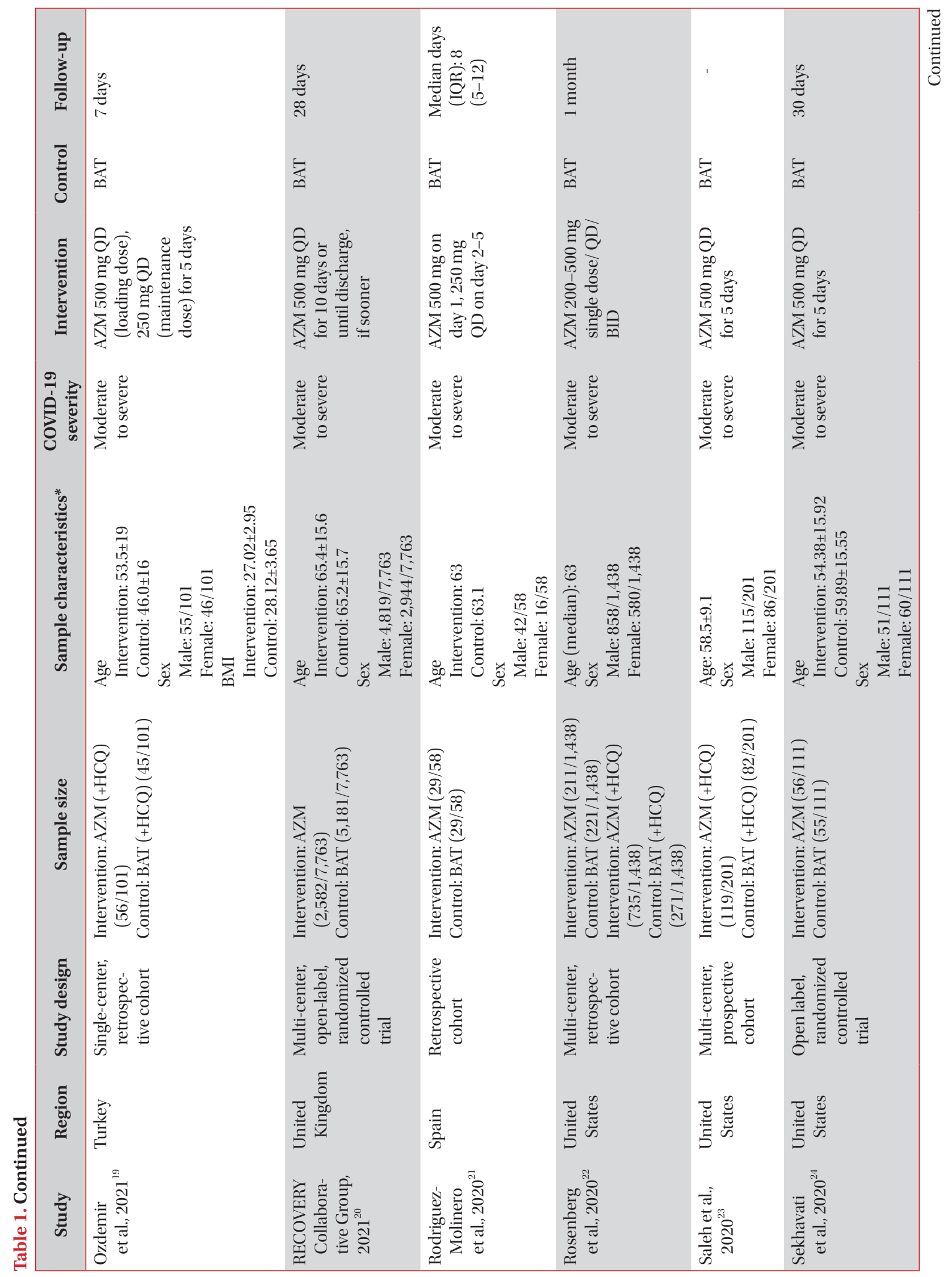




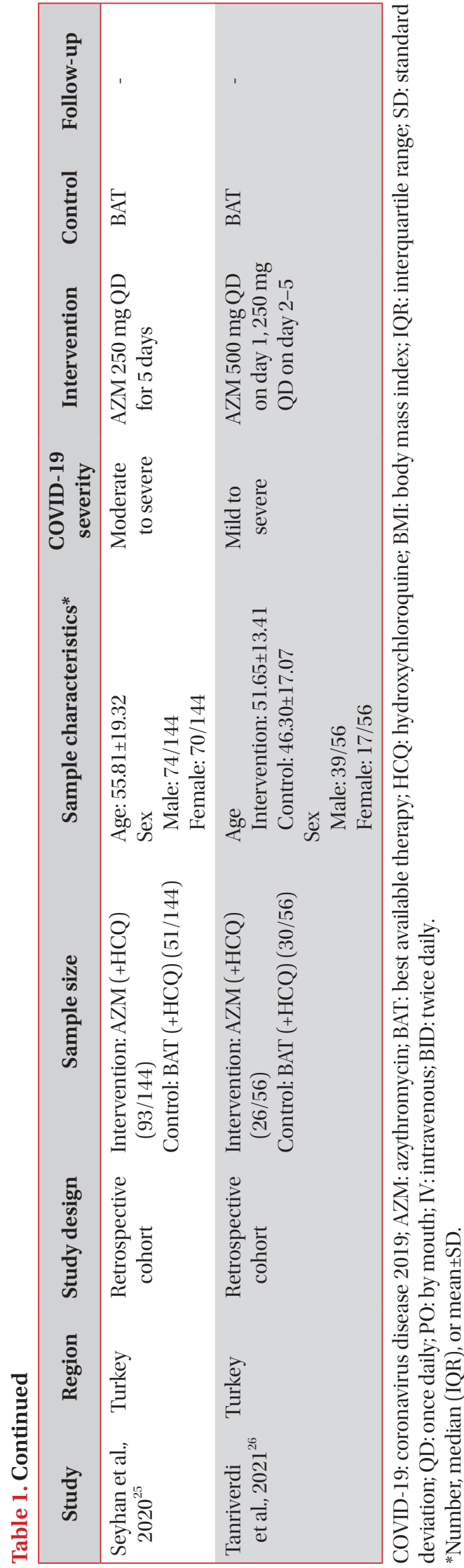

World Health Organization. There was no restriction for age, races, occupation, economy/social status, religion, country, or underlying condition.

\section{3) Intervention}

Studies evaluating all types of azithromycin for the treatment of COVID-19 were included in this study. Azithromycin was given in any dosage regimen either alone or in combination with the best available therapy (BAT).

\section{4) Comparison and outcome}

Comparators included patients treated with placebo and/or only given BAT. Outcomes of interest were efficacy and safety of azithromycin in COVID-19 treatment. Efficacy included clinical improvement, hospitalization period, and mortality. Safety included toxicity and serious adverse events occurring during treatment.

\section{Search strategy and study selection}

Literature search was carried out with multiple electronic databases such as PubMed, ScienceDirect, EBSCO, and ProQuest from inception to March 2021. No time and language restriction were applied. This study only included peerreviewed articles of clinical trials evaluating the efficacy and safety of azithromycin in COVID-19 patients. The search was performed by three independent reviewers (GM, G, and N).

Articles were identified using keywords ("COVID-19" OR "COVID-19" OR "2019 novel coronavirus disease" OR "Coronavirus disease 2019" OR "COVID19" OR "2019 nCoV disease" OR "SARS-CoV-2 infection") AND ("azithromycin") with their respective Medical Subject Headings (MeSH) terms, if applicable. After removing duplicates using EndNote program, retrieved articles were screened based on their titles and abstracts. Thereafter, potentially eligible full-text articles were thoroughly assessed using the eligibility criteria described above. Any emerging discrepancies were resolved by consensus among the three reviewers.

\section{Data extraction}

The following data were extracted from these studies: (1) first author, (2) publication year, (3) region, (4) study design, (5) sample characteristics and size, (6) COVID-19 severity, (7) intervention (dose, route of administration, duration, other treatments besides azithromycin) and control, (8) follow-up period, if any, and (9) efficacy and safety of azithromycin.

\section{Quality assessment and reliability of data}

Version 2 of the Cochrane Risk of Bias tool (RoB-2) was used to assess the quality of included randomized trials ${ }^{29}$. Newcastle-Ottawa Scale was used to evaluate the quality of 


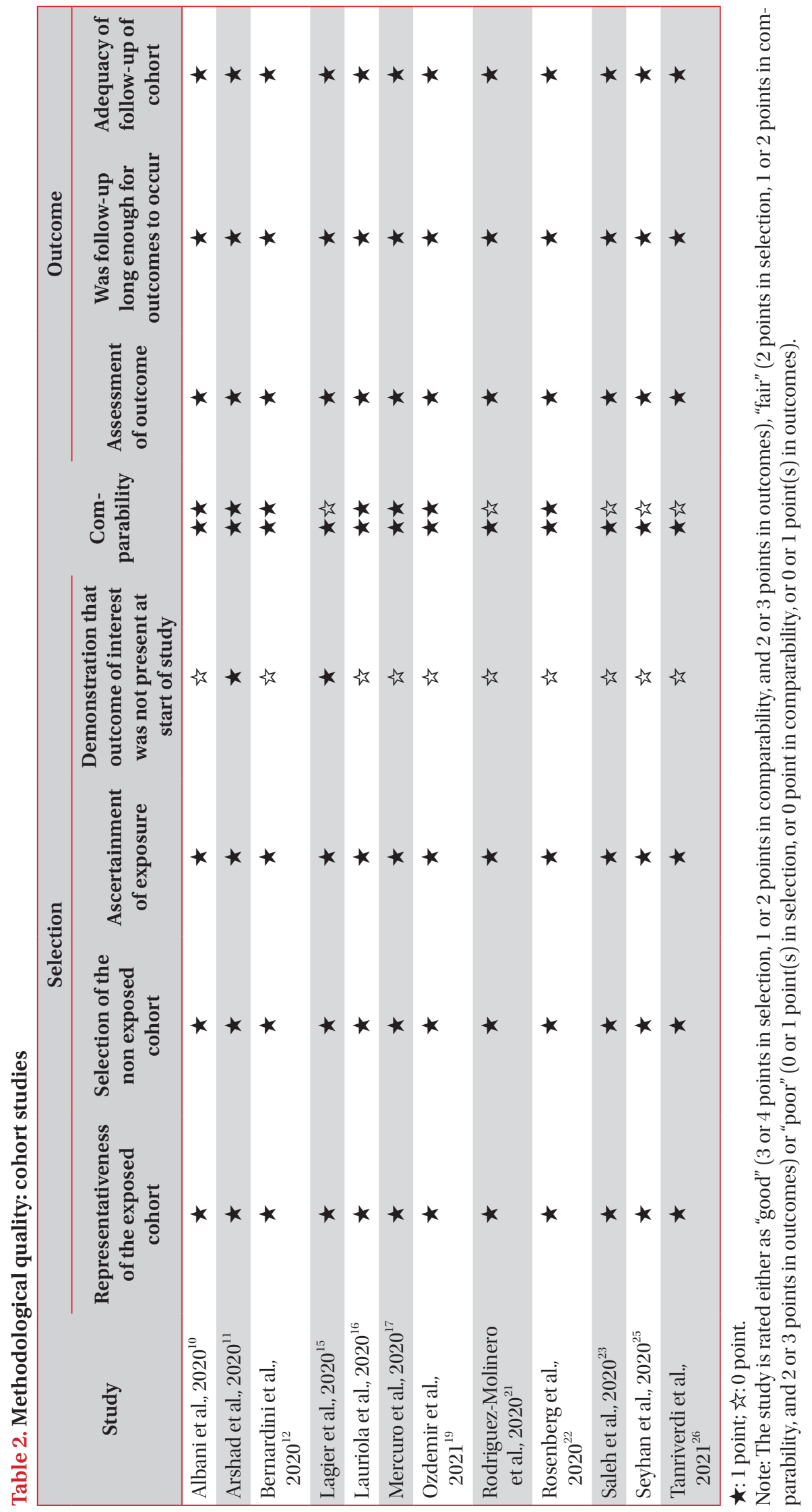


non-randomized study design for the included study ${ }^{30}$. Three researchers (G, GM, and N) independently evaluated whether a study had low or some concerns or high risk of bias. Any discrepancies were resolved through discussion. Trial sequential analysis (TSA) was performed to determine the required sample size and confirm whether the meta-analysis was conclusive. TSA generated thresholds for declaring significance of the result to avoid an overestimation of intervention effects and prevent spurious results. A two-sided trial of the sequential monitoring boundary type was used in our TSA. The required information size was calculated with $\alpha=0.05$. TSA was performed using TSA version 0.9.5.10 beta $^{31}$.

\section{Data synthesis and statistical analysis}

Either odds ratio (OR) or weighted mean difference with a

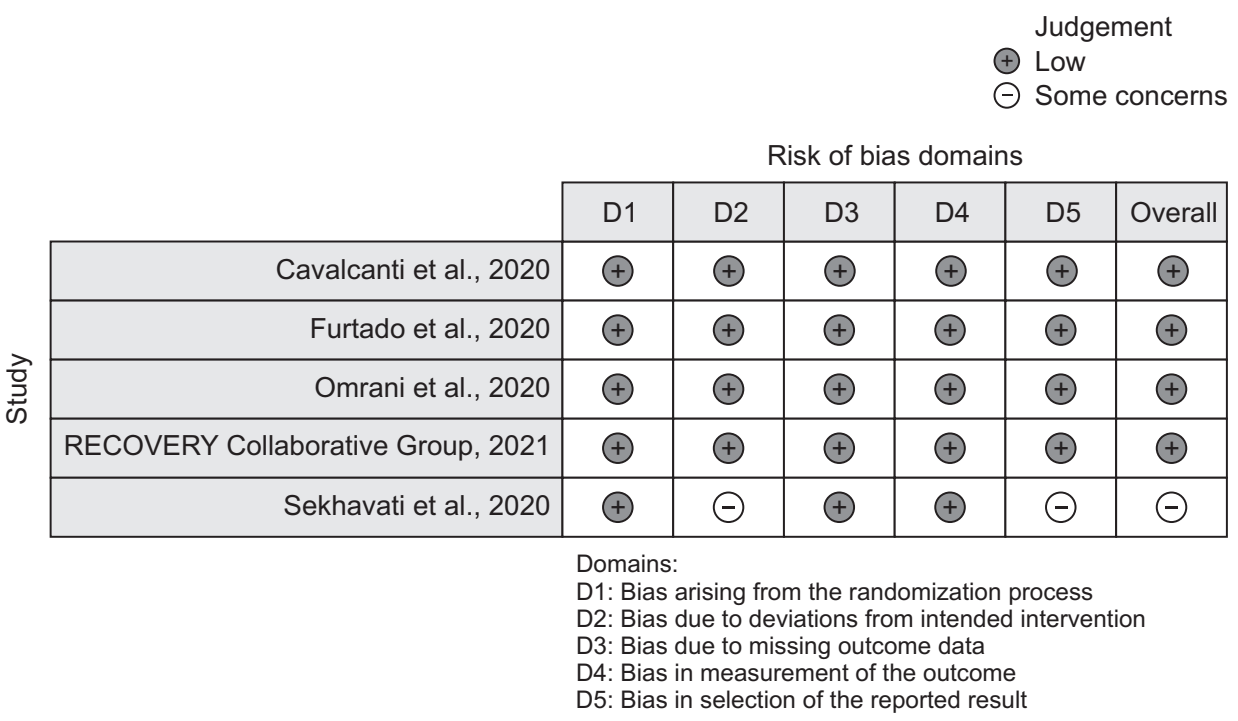

Figure 2. Methodological quality: randomized controlled trials.

\begin{tabular}{|c|c|c|c|c|c|c|c|c|c|c|}
\hline \multirow{3}{*}{$\begin{array}{l}\text { A Mortality rate } \\
\text { Study or subgroup } \\
\text { Albani et al. }\end{array}$} & \multicolumn{2}{|c|}{ Azithromycin } & \multicolumn{2}{|c|}{ Control } & \multirow{3}{*}{$\begin{array}{c}\text { Weight } \\
10.9 \%\end{array}$} & \multirow{3}{*}{$\begin{array}{c}\begin{array}{c}\text { Odds ratio } \\
\mathrm{M}-\mathrm{H}, \text { random, } 95 \% \mathrm{Cl}\end{array} \\
0.49[0.36,0.67]\end{array}$} & \multirow{2}{*}{\multicolumn{4}{|c|}{$\begin{array}{c}\text { Odds ratio } \\
\mathrm{M}-\mathrm{H} \text {, random, } 95 \% \mathrm{Cl}\end{array}$}} \\
\hline & \multirow{2}{*}{$\begin{array}{r}\text { Events } \\
69\end{array}$} & \multirow{2}{*}{$\begin{array}{c}\text { Total } \\
421\end{array}$} & \multirow{2}{*}{$\begin{array}{r}\text { Events } \\
172\end{array}$} & \multirow{2}{*}{$\begin{array}{r}\text { Total } \\
605\end{array}$} & & & & & & \\
\hline & & & & & & & & & & \\
\hline Albani et al. (+HCQ) & 53 & 166 & 60 & 211 & $9.0 \%$ & $1.18[0.76,1.84]$ & & & - & \\
\hline Arshad et al. & 33 & 147 & 108 & 409 & $8.9 \%$ & $0.81[0.52,1.26]$ & & & . & \\
\hline Arshad et al. $(+\mathrm{HCQ})$ & 157 & 783 & 162 & 1,202 & $11.9 \%$ & $1.61[1.27,2.05]$ & & & - & \\
\hline Bernardini et al. & 9 & 53 & 8 & 40 & $3.4 \%$ & $0.82[0.28,2.35]$ & & & & \\
\hline Cavalcanti et al. & 5 & 217 & 9 & 221 & $3.2 \%$ & $0.56[0.18,1.69]$ & & & - & \\
\hline Furtado et al. & 66 & 214 & 55 & 183 & $9.2 \%$ & $1.04[0.68,1.59]$ & & & - & \\
\hline Lagier et al. & 5 & 137 & 4 & 162 & $2.3 \%$ & $1.50[0.39,5.69]$ & & & & \\
\hline Lagier et al. (+HCQ) & 8 & 218 & 2 & 101 & $1.8 \%$ & $1.89[0.39,9.04]$ & & & & \\
\hline Lagier et al. (+HCQ) & 16 & 3,119 & 2 & 101 & $2.0 \%$ & $0.26[0.06,1.13]$ & & & & \\
\hline Lauriola et al. & 102 & 297 & 7 & 17 & $3.7 \%$ & $0.75[0.28,2.02]$ & & & - & \\
\hline RECOVERY Collaborative Group & 561 & 2,582 & 1,162 & 5,181 & $13.3 \%$ & $0.96[0.86,1.08]$ & & & & \\
\hline Rodriguez-Molinero et al. & 1 & 29 & 2 & 29 & $0.8 \%$ & $0.48[0.04,5.63]$ & & & & \\
\hline Rosenberg et al. & 21 & 211 & 28 & 221 & $6.9 \%$ & $0.76[0.42,1.39]$ & & & - & \\
\hline Rosenberg et al. (+HCQ) & 189 & 735 & 54 & 271 & $10.5 \%$ & $1.39[0.99,1.96]$ & & & -- & \\
\hline Sekhavati et al. & 0 & 56 & 1 & 55 & $0.5 \%$ & $0.32[0.01,8.06]$ & & & & \\
\hline Tanriverd et al. & 5 & 26 & 3 & 30 & $1.8 \%$ & $2.14[0.46,10.00]$ & & & & \\
\hline Total $(95 \% \mathrm{Cl})$ & & 9,411 & & 9,039 & $100.0 \%$ & $0.95[0.76,1.19]$ & & & & \\
\hline Total events & 1,300 & & 1,839 & & & & & & & \\
\hline Heterogeneity: $\operatorname{Tau}^{2}=0.10 ; \mathrm{Chi}^{2}=48$ & $d f=16(p$ & $.0001)$ & ${ }^{2}=67 \%$ & & & & 0.01 & 0.1 & 10 & 100 \\
\hline Test for overall effect: $Z=0.44(p=0$ & & & & & & & Favc & [Azith & Favours [ & [Control] \\
\hline
\end{tabular}

Figure 3. (A-D) Efficacy of azithromycin. The horizontal line indicates 95\% CI of the study. The square represents the result of each individual study. The size of the square varies according to the weight of a particular study. The diamond at the bottom of the plot represents pooled analysis of all included studies. Outer edges of the diamond indicate CIs. CI: confidence interval; df: degree of freedom; $\mathrm{I}^{2}$ : test of heterogeneity; M-H: Mantel-Haenszel. 


\begin{tabular}{|c|c|c|c|c|c|c|c|c|c|}
\hline \multirow{2}{*}{$\begin{array}{l}\text { B Respiratory support } \\
\text { Study or subgroup }\end{array}$} & \multicolumn{2}{|c|}{ Azithromycin } & \multicolumn{2}{|c|}{ Control } & \multirow[b]{2}{*}{ Weight } & \multirow{2}{*}{$\begin{array}{c}\text { Odds ratio } \\
\mathrm{M}-\mathrm{H}, \text { random, } 95 \% \mathrm{Cl}\end{array}$} & \multirow{2}{*}{\multicolumn{3}{|c|}{$\begin{array}{c}\text { Odds ratio } \\
\mathrm{M}-\mathrm{H} \text {, random, } 95 \% \mathrm{Cl}\end{array}$}} \\
\hline & Events & Total & Events & Total & & & & & \\
\hline \multicolumn{10}{|l|}{ 1.16.1 Oxygen supplementation } \\
\hline Arshad et al. & 14 & 147 & 34 & 409 & $6.9 \%$ & $1.16[0.60,2.23]$ & & - & \\
\hline Arshad et al. (+HCQ) & 234 & 783 & 166 & 1,202 & $10.1 \%$ & $2.66[2.13,3.33]$ & & $\rightarrow$ & \\
\hline Cavalcanti et al. & 5 & 217 & 6 & 221 & $3.7 \%$ & $0.85[0.25,2.81]$ & & - & \\
\hline Furtado et al. & 21 & 214 & 9 & 183 & $5.8 \%$ & $2.10[0.94,4.72]$ & & & \\
\hline Mercuro et al. & 40 & 53 & 24 & 37 & $5.1 \%$ & $1.67[0.66,4.18]$ & & & \\
\hline Subtotal $(95 \% \mathrm{Cl})$ & & 1,414 & & 2,052 & $31.6 \%$ & $1.77[1.13,2.77]$ & & & \\
\hline Total events & 314 & & 239 & & & & & & \\
\hline \multicolumn{10}{|c|}{ Heterogeneity: $\mathrm{Tau}^{2}=0.13 ; \mathrm{Chi}^{2}=9.01, \mathrm{df}=4(\mathrm{p}=0.06) ; \mathrm{l}^{2}=56 \%$} \\
\hline \multicolumn{10}{|l|}{ Test for overall effect: $Z=2.50(p=0.01)$} \\
\hline \multicolumn{10}{|l|}{ 1.16.2 NIV/HFNC } \\
\hline Cavalcanti et al. & 17 & 217 & 19 & 221 & $6.7 \%$ & $0.90[0.46,1.79]$ & & & \\
\hline Furtado et al. & 5 & 214 & 3 & 183 & $2.9 \%$ & $1.44[0.44,6.09]$ & & & \\
\hline RECOVERY Collaborative Group & 214 & 1,368 & 467 & 2,705 & $10.4 \%$ & $0.89[0.74,1.06]$ & & & \\
\hline Subtotal $(95 \% \mathrm{Cl})$ & & 1,799 & & 3,109 & $19.9 \%$ & $0.90[0.76,1.06]$ & & & \\
\hline Total events & 236 & & 489 & & & & & & \\
\hline \multicolumn{10}{|c|}{ Heterogeneity: $\operatorname{Tau}^{2}=0.00 ; \mathrm{Ch}^{2}=0.42, \mathrm{df}=2(p=0.81) ; \mathrm{I}^{2}=0 \%$} \\
\hline \multicolumn{10}{|l|}{ Test for overall effect: $Z=1.27(p=0.20)$} \\
\hline \multicolumn{10}{|l|}{ 1.16.3 Mechanical ventilation/ECMO } \\
\hline Cavalcanti et al. & 20 & 217 & 16 & 221 & $6.7 \%$ & $1.30[0.66,2.58]$ & & & \\
\hline Furtado et al. & 69 & 214 & 52 & 183 & $8.7 \%$ & $1.20[0.78,1.84]$ & & & \\
\hline Lauriola et al. & 73 & 297 & 3 & 17 & $3.4 \%$ & $1.52[0.43,5.44]$ & & & \\
\hline Mercuro et al. & 16 & 53 & 7 & 37 & $4.6 \%$ & $1.85[0.67,5.09]$ & & & \\
\hline RECOVERY Collaborative Group & 57 & 1,368 & 115 & 2,705 & $9.5 \%$ & $0.98[0.71,1.35]$ & & & \\
\hline Rosenberg et al. & 13 & 211 & 18 & 221 & $6.3 \%$ & $0.74[0.35,1.55]$ & & & \\
\hline Rosenberg et al. (+HCQ) & 199 & 735 & 51 & 271 & $9.3 \%$ & $1.60[1.13,2.26]$ & & & \\
\hline Subtotal $(95 \% \mathrm{Cl})$ & & 3,095 & & 3,655 & $48.4 \%$ & $1.22[0.99,1.49]$ & & & \\
\hline Total events & 447 & & 262 & & & & & & \\
\hline \multicolumn{10}{|c|}{ Heterogeneity: $\operatorname{Tau}^{2}=0.01 ; \mathrm{Chi}^{2}=6.71, \mathrm{df}=6(\mathrm{p}=0.35) ; \mathrm{I}^{2}=11 \%$} \\
\hline \multicolumn{10}{|l|}{ Test for overall effect: $Z=1.86(p=0.06)$} \\
\hline Total $(95 \% \mathrm{Cl})$ & & 6,308 & & 8,816 & $100.0 \%$ & $1.30[0.98,1.73]$ & & & \\
\hline Total events & 997 & & 990 & & & & & & \\
\hline \multicolumn{7}{|c|}{ Heterogeneity: $\operatorname{Tau}^{2}=0.20 ; \mathrm{Chi}^{2}=67.57, \mathrm{df}=14(p<0.00001) ; \mathrm{I}^{2}=79 \%$} & $0.02 \quad 0.1$ & & 10 \\
\hline \multicolumn{7}{|c|}{ Test for overall effect: $Z=1.80(p=0.07)$} & Favours $[\mathrm{Az}$ & Favc & [Control] \\
\hline
\end{tabular}

Figure 3. Continued.

confidence interval (CI) of 95\% was used to determine the efficacy and safety of azithromycin in COVID-19 patients. Either fixed-effects or random-effects model was used depending on the study heterogeneity. Heterogeneity of included studies was assessed using Cochrane's Q test of homogeneity and Higgins $I^{2}$ statistics. Subgroup analysis was conducted to find the possible cause of heterogeneity.

Funnel plot was used to assess publication bias visually. Asymmetric funnel plot indicated possible publication bias. Begg and Mazumdar rank correlation test and Egger's test of the intercept were used to determine the presence of publication bias statistically. All statistical tests were performed using Review Manager (RevMan) 5.3 and MedCalc version 19.5. $1^{32,33}$.

\section{Confidence in cumulative evidence}

Grading of Recommendations, Assessment, Development, and Evaluations (GRADE) was performed to determine the confidence in cumulative evidence. Judgement was made considering the presence of study limitations, consistency, directness, imprecision, and/or reporting bias. Overall certainty of evidence was shown as high, moderate, low, or very low.

\section{Results}

\section{Search results}

After searching electronic databases, 2,733 studies were found. After screening titles and abstracts, 1,889 articles were 


\begin{tabular}{|c|c|c|c|c|c|c|c|c|c|c|c|c|}
\hline \multirow{2}{*}{$\begin{array}{l}\text { C Hospitalization period } \\
\text { Study or subgroup }\end{array}$} & \multicolumn{3}{|c|}{ Azithromycin } & \multicolumn{3}{|c|}{ Control } & \multirow[b]{2}{*}{ Weight } & \multirow{2}{*}{$\begin{array}{l}\text { Std. Mean difference } \\
\text { IV, random, } 95 \% \mathrm{Cl}\end{array}$} & \multirow{2}{*}{\multicolumn{3}{|c|}{$\begin{array}{l}\text { Std. Mean difference } \\
\mathrm{IV} \text {, random, } 95 \% \mathrm{Cl}\end{array}$}} & \\
\hline & Mean & SD & $\overline{\text { Total }}$ & $\overline{\text { Mean }}$ & SD & Total & & & & & & \\
\hline Albani et al. & 6 & 4.4 & 421 & 6 & 3.7 & 605 & $7.3 \%$ & $0.00[-0.12,0.12]$ & & & & \\
\hline Albani et al. (+HCQ) & 10 & 8.1 & 166 & 10 & 7.4 & 211 & $6.7 \%$ & $0.00[-0.20,0.20]$ & & & & \\
\hline Arshad et al. & 5.3 & 4.5 & 147 & 5.6 & 4.8 & 409 & $6.8 \%$ & $-0.06[-0.25,0.13]$ & & & & \\
\hline Arshad et al. $(+\mathrm{HCQ})$ & 10.7 & 7.5 & 783 & 8 & 5.8 & 1,202 & $7.5 \%$ & $0.41[0.32,0.50]$ & & & - & \\
\hline Cavalcanti et al. & 9.4 & 7.8 & 217 & 8.9 & 6.2 & 221 & $6.8 \%$ & $0.07[-0.12,0.26]$ & & & & \\
\hline Furtado et al. & 26 & 13.3 & 214 & 18 & 13.3 & 183 & $6.7 \%$ & $0.60[0.40,0.80]$ & & & - & \\
\hline Lagier et al. & 8.8 & 7.1 & 137 & 7.5 & 6.9 & 162 & $6.4 \%$ & $0.19[-0.04,0.41]$ & & & & \\
\hline Lagier et al. (+HCQ) & 11.8 & 9.8 & 218 & 5.7 & 4 & 101 & $6.3 \%$ & $0.72[0.48,0.97]$ & & & - & \\
\hline Lagier et al. (+HCQ) & 7.3 & 7 & 3,119 & 5.7 & 4 & 101 & $6.7 \%$ & $0.23[0.03,0.43]$ & & & & \\
\hline Lauriola et al. & 14.9 & 9.8 & 297 & 6.8 & 4.6 & 17 & $4.0 \%$ & $0.84[0.35,1.33]$ & & & & \\
\hline Mercuro et al. & 6.5 & 4.3 & 53 & 5 & 3 & 37 & $4.6 \%$ & $0.39[-0.03,0.81]$ & & & 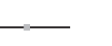 & \\
\hline RECOVERY Collaborative Group & 10 & 17 & 2,582 & 11 & 17 & 5,181 & $7.6 \%$ & $-0.06[-0.11,-0.01]$ & & & & \\
\hline Rosenberg et al. & 3 & 2.2 & 211 & 4 & 3.7 & 221 & $6.8 \%$ & $-0.33[-0.52,-0.14]$ & & - & & \\
\hline Rosenberg et al. (+HCQ) & 7 & 4.4 & 735 & 7 & 5.9 & 271 & $7.2 \%$ & $0.00[-0.14,0.14]$ & & & & \\
\hline Sekhavati et al. & 4.61 & 2.59 & 56 & 5.96 & 3.21 & 55 & $5.0 \%$ & $-0.46[-0.84,-0.08]$ & & & & \\
\hline Tanriverd et al. & 6.68 & 2.23 & 26 & 8.16 & 2.56 & 30 & $3.7 \%$ & $-0.60[-1.14,-0.07]$ & & & & \\
\hline Total $(95 \% \mathrm{Cl})$ & & & 9,382 & & & 9,007 & $100.0 \%$ & $0.12[-0.02,0.27]$ & & & & \\
\hline \multirow{2}{*}{\multicolumn{9}{|c|}{$\begin{array}{l}\text { Heterogeneity: } \text { Tau }^{2}=0.07 ; \mathrm{Chi}^{2}=187.35, \mathrm{df}=15(p<0.00001) ; I^{2}=92 \% \\
\text { Test for overall effect: } Z=1.70(p=0.09)\end{array}$}} & & & & \\
\hline & & & & & & & & & 2 & -1 & 1 & 2 \\
\hline
\end{tabular}

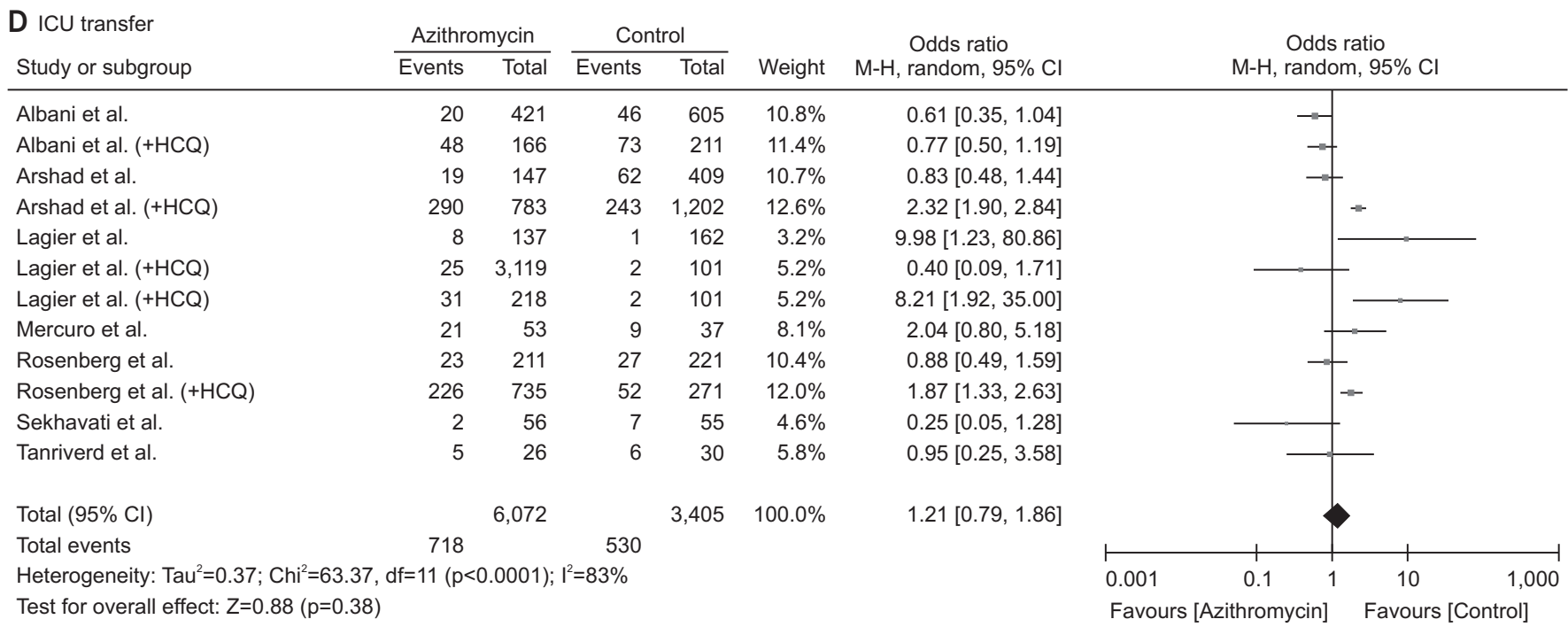

Figure 3. Continued.

found, of which 104 were assessed for eligibility. A total of 17 studies were included in the meta-analysis finally ${ }^{10-26}$. Search flowchart and selection methods used in this study are summarized in Figure 1.

\section{Characteristics of included studies}

Included studies were conducted in various regions, including America ${ }^{11,13,14,17,22-24}$, Europe ${ }^{10,12,15,16,20,21}$, and Middle East $^{18,19,25,26}$. All studies recruited adults aged 45 to 83 years. Included patients had common underlying conditions such as hypertension, diabetes mellitus, chronic obstructive pul- monary disease, and cardiovascular disease. The severity of COVID-19 ranged from mild to severe. Overall, azithromycin was given as much as $250-500 \mathrm{mg}$ daily for $5-10$ days. Other treatments besides azithromycin that the majority of patients received were glucocorticoids, hydroxychloroquine, diuretics, and anticoagulants. All four randomized controlled trials had low risk of bias except that one study showed some concerns of bias in classifying the interventions and measurements of outcomes $^{24}$. Twelve cohort studies showed good quality in terms of selection, comparability, and outcomes. Characteristics of included studies are summarized in Tables 1, 2 and Figure 2. 
A Secondary infection

\begin{tabular}{|c|c|c|c|c|c|c|}
\hline \multirow{2}{*}{$\begin{array}{l}\text { A Secondary infection } \\
\text { Study or subgroup }\end{array}$} & \multicolumn{2}{|c|}{ Azithromycin } & \multicolumn{2}{|c|}{ Control } & \multirow[b]{2}{*}{ Weight } & \multirow{2}{*}{$\begin{array}{c}\text { Odds ratio } \\
\text { M-H, fixed, } 95 \% \text { C }\end{array}$} \\
\hline & Events & Total & Events & Total & & \\
\hline Cavalcanti et al. & 0 & 217 & 1 & 221 & $3.3 \%$ & $0.34[0.01,8.34]$ \\
\hline Furtado et al. & 87 & 214 & 65 & 183 & $92.4 \%$ & $1.24[0.83,1.87]$ \\
\hline Lagier et al. & 1 & 3,337 & 0 & 101 & $2.2 \%$ & $0.09[0.00,2.25]$ \\
\hline Omrani et al. & 3 & 152 & 1 & 152 & $2.2 \%$ & $3.04[0.31,29.56]$ \\
\hline Total $(95 \% \mathrm{Cl})$ & & 3,920 & & 657 & $100.0 \%$ & $1.23[0.83,1.82]$ \\
\hline Total events & 91 & & 67 & & & \\
\hline
\end{tabular}

Heterogeneity: $C i^{2}=3.76, d f=3(p=0.29) ; I^{2}=20 \%$

Test for overall effect: $Z=1.02(p=0.31)$

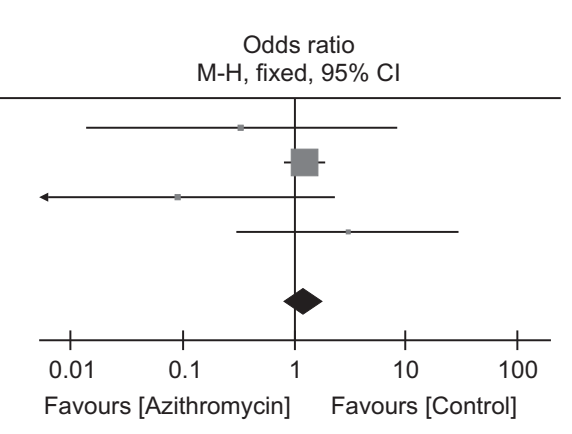

B Hypoglycemia

Study or subgroup

$\frac{\text { Azithromycin }}{\text { Events Total }}$

Cavalcanti et al. 2020

Rosenberg et al. 2020

Rosenberg et al. 2020 (+HCQ)

Total $(95 \% \mathrm{Cl})$

Total events

Heterogeneity: $\mathrm{Chi}^{2}=2.76, \mathrm{df}=2(\mathrm{p}=0.25) ; \mathrm{I}^{2}=28 \%$

Test for overall effect: $Z=0.96(p=0.34)$

$\begin{array}{rrrrrr}0 & 217 & 1 & 221 & 7.4 \% & 0.34[0.01,8.34] \\ 1 & 211 & 6 & 221 & 29.1 \% & 0.17[0.02,1.43] \\ 25 & 735 & 9 & 271 & 63.5 \% & 1.03[0.47,2.22] \\ & & & & & \\ & 1,163 & & 713 & 100.0 \% & 0.73[0.38,1.40]\end{array}$

16
Odds ratio Odds ratio $\mathrm{M}-\mathrm{H}$, fixed, $95 \% \mathrm{Cl}$

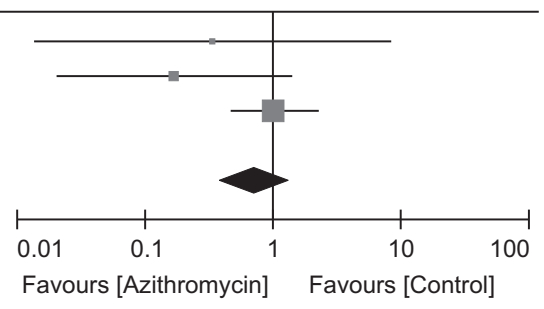

C Gastrointestinal symptoms

Study or subgroup

Azithromycin Control

\begin{tabular}{llll} 
Azithromycin & \multicolumn{2}{c}{ Control } & Odds ratio \\
Events Total & Events Total & Weight & $\mathrm{M}-\mathrm{H}$, random, $95 \% \mathrm{Cl}$
\end{tabular}

Odds ratio

$\mathrm{M}-\mathrm{H}$, random, $95 \% \mathrm{Cl}$

\title{
2.2.1 Diarrhea
}

Lagier et al.

Lagier et al. (+HCQ)

Rosenberg et al.

Rosenberg et al. (+HCQ)

Subtotal $(95 \% \mathrm{Cl})$

Events 137

$\begin{array}{lc}\text { Total events } & 155 \\ \text { Heterogeneity: } \text { Tau }^{2}=0.00 ; \mathrm{Chi}^{2}=1.19, \mathrm{df}=3(\mathrm{p}=0.76) ; \mathrm{I}^{2}=0 \%\end{array}$

$\begin{array}{rrrrr}0 & 137 & 1 & 162 & 1.1 \% \\ 54 & 3,337 & 1 & 101 & 3.0 \% \\ 16 & 211 & 16 & 221 & 22.8 \% \\ 85 & 735 & 22 & 271 & 48.9 \% \\ & 4,420 & & 755 & 75.8 \%\end{array}$

$0.39[0.02,9.69]$

$1.64[0.23,12.01]$

$1.05[0.51,2.16]$

$1.48[0.91,2.42]$

$1.31[0.89,1.95]$

Test for overall effect: $Z=1.36(p=0.17)$

\author{
2.2.2 Nausea/vomiting \\ Cavalcanti et al. \\ Lagier et al. (+HCQ) \\ Subtotal $(95 \% \mathrm{Cl})$ \\ Total events

\subsubsection{Others \\ Cavalcanti et al. \\ Lagier et al. \\ Lagier et al. (+HCQ) \\ Subtotal $(95 \% \mathrm{Cl})$} \\ Total events
}

$\begin{array}{rr}6 & 217 \\ 26 & 3,337 \\ & 3,554\end{array}$

$\begin{array}{rrr}9 & 221 & 10.7 \% \\ 2 & 101 & 5.6 \% \\ & 322 & 16.3 \%\end{array}$

32

11

$0.67[0.23,1.91]$

$0.39[0.09,1.66]$

$0.56[0.24,1.30]$

Heterogeneity: $\operatorname{Tau}^{2}=0.00 ; \mathrm{Chi}^{2}=0.37, \mathrm{df}=1 \quad(\mathrm{p}=0.54) ; \mathrm{I}^{2}=0 \%$

Test for overall effect: $Z=1.35(p=0.18)$

Heterogeneity: $\operatorname{Tau}^{2}=0.00 ; \mathrm{Chi}^{2}=0.00, \mathrm{df}=2(\mathrm{p}=1.00) ; \mathrm{l}^{2}=0 \%$

Test for overall effect: $Z=1.63(p=0.10)$

\section{Subtotal $(95 \% \mathrm{Cl})$}

Total events

11,665 211
$\begin{array}{ll}0 & 217\end{array}$
0
137
$24 \quad 3,337$
3,691

24

Heterogeneity: $\operatorname{Tau}^{2}=0.00 ; \mathrm{Chi}^{2}=7.93, \mathrm{df}=8(\mathrm{p}=0.44) ; \mathrm{l}^{2}=0 \%$

Test for overall effect: $Z=0.18(p=0.86)$

Test for subgroup differences: $\mathrm{Chi}^{2}=6.31, \mathrm{df}=2(\mathrm{p}=0.04) ; \mathrm{l}^{2}=68.3 \%$

$\begin{array}{lll}1 & 221 & 1.1 \% \\ 1 & 162 & 1.1 \% \\ 2 & 101 & 5.6 \% \\ & 484 & 7.9 \% \\ 4 & & \end{array}$

$0.34[0.01,8.34]$

$0.39[0.02,9.69]$

$0.36[0.08,1.54]$

$0.36[0.11,1.23]$

$1.03[0.73,1.45]$ 55

$1,561 \quad 100.0 \%$

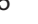

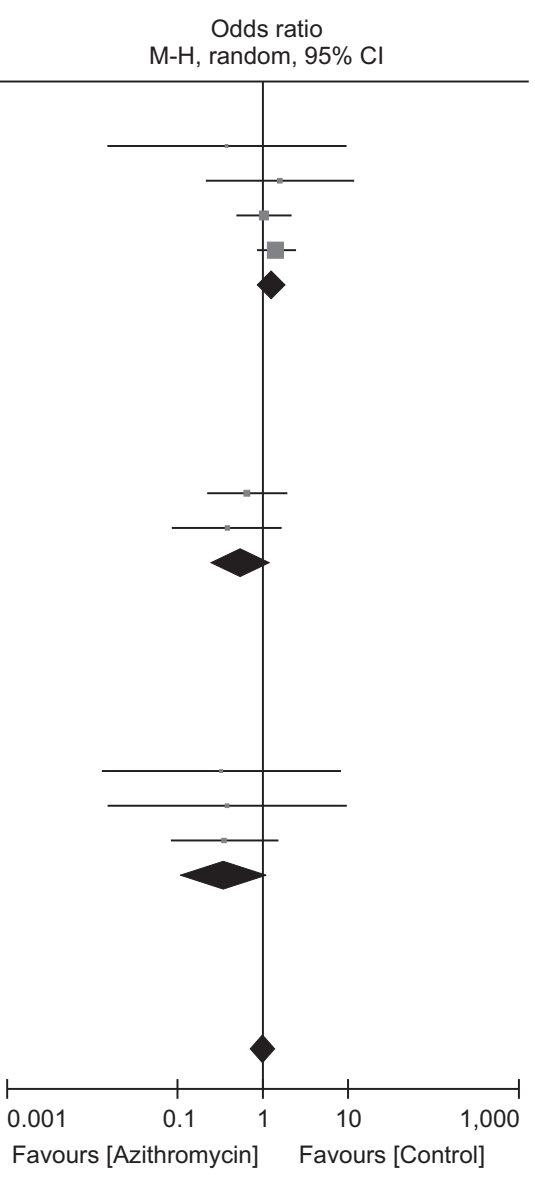

Figure 4. (A-D) Safety of azithromycin. The square represents the result of each individual study. The size of the square varies according to the weight of a particular study. The diamond at the bottom of the plot represents pooled analysis of all included studies. Outer edges of the diamond indicate CIs. CI: confidence interval; df: degree of freedom; $I^{2}$ : test of heterogeneity; M-H: Mantel-Haenszel. 


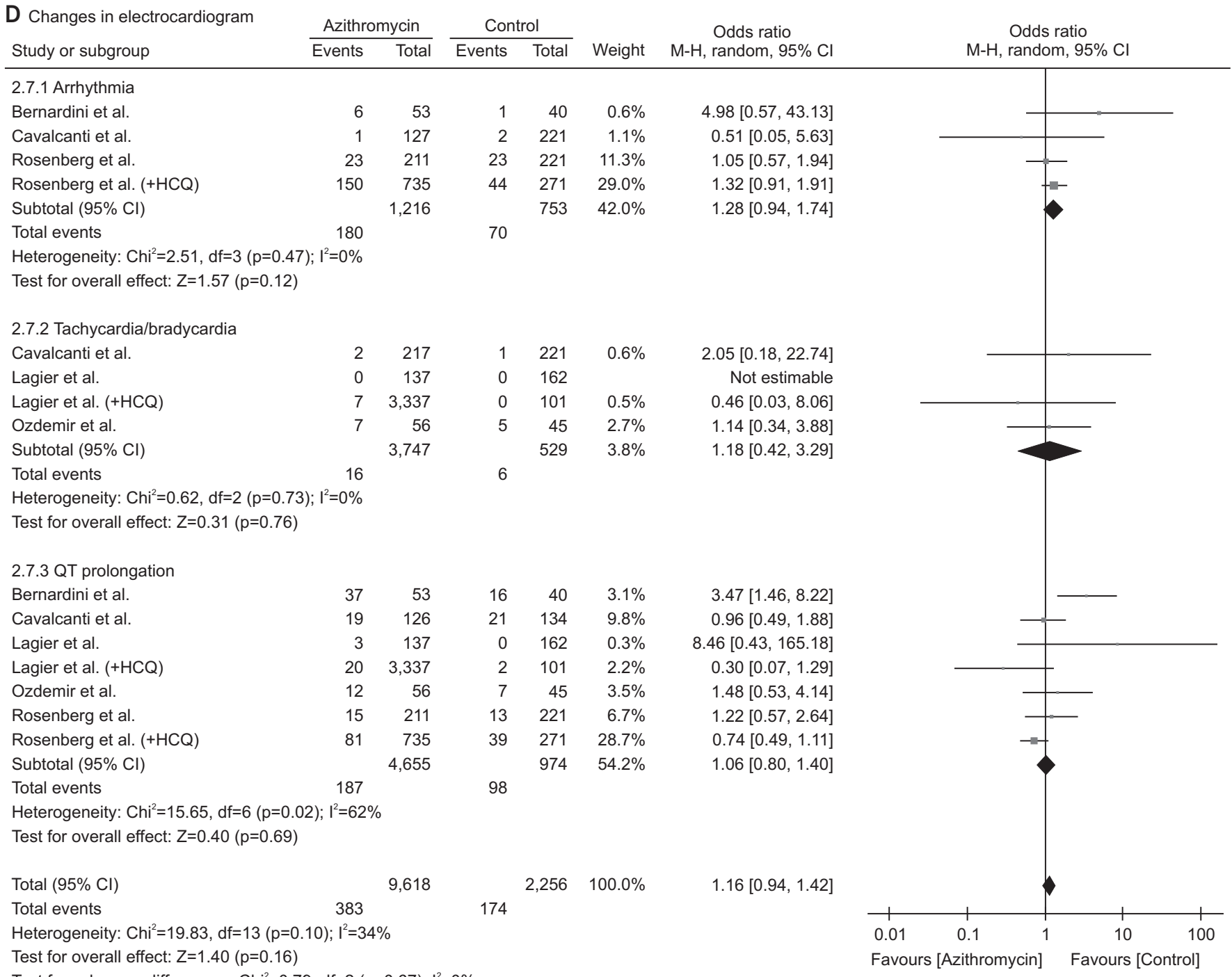

Figure 4. Continued.

\section{Meta-analysis: efficacy and safety of azithromycin in COVID-19 patients}

COVID-19 patients treated with azithromycin showed lower mortality rate than controls, although the difference between the two was not statistically significant (OR, 0.95; 95\% CI, 0.76-1.19; $\mathrm{p}=0.66 ; \mathrm{I}^{2}=67 \%$ ) (Figure $3 \mathrm{~A}$ ). Needs for oxygen supplementation (OR, 1.77; 95\% CI, 1.13-2.77) and mechanical ventilation/extracorporeal membrane oxygenation (OR, 1.22; 95\% CI, 0.99-1.49) were higher for patients treated with azithromycin, although the overall need for respiratory support did not significantly differ between the two groups (OR, 1.30; 95\% CI, 0.98-1.73; $\mathrm{p}=0.07 ; \mathrm{I}^{2}=79 \%$ ) (Figure 3B). Azithromycin-treated patients showed a longer hospitalization period (standardized mean difference, $0.12 ; 95 \% \mathrm{CI},-0.02$ to $0.27 ; \mathrm{p}=0.09 ; \mathrm{I}^{2}=92 \%$ ) (Figure $3 \mathrm{C}$ ) and a higher ICU transfer
(OR, 1.21; 95\% CI, 0.79-1.86; $\mathrm{p}=0.38 ; \mathrm{I}^{2}=83 \%$ ) (Figure 3D) compared to the control group, although differences between the two groups were not statistically significant. Interestingly, this meta-analysis showed that patients receiving both azithromycin and hydroxychloroquine had a higher mortality rate $(p=0.03)$ and more likely to need respiratory support $(p=0.01)$ compared to those receiving azithromycin only $(\mathrm{OR}, 1.21 ; 95 \%$ CI, $0.92-1.59$ vs. OR, $0.80 ; 95 \%$ CI, $0.61-1.05$ and $\mathrm{OR}, 1.59$; $95 \%$ CI, $1.13-2.24$ vs. OR, $0.98 ; 95 \%$ CI, $0.84-1.15$, respectively).

Azithromycin treatment did not significantly increase the risk of getting secondary infection $(\mathrm{OR}, 1.23$; 95\% CI, 0.83-1.82; $\mathrm{p}=0.31 ; \mathrm{I}^{2}=20 \%$ ) (Figure $4 \mathrm{~A}$ ) or hypoglycemia (OR, $0.73 ; 95 \%$ CI, 0.38-1.40; $\mathrm{p}=0.34 ; \mathrm{I}^{2}=28 \%$ ) (Figure $4 \mathrm{~B}$ ). No significant difference was observed in gastrointestinal symptoms between the two groups (OR, 1.03; 95\% CI, 0.73-1.45; $\mathrm{p}=0.86 ; \mathrm{I}^{2}=0 \%$ ) (Figure $4 \mathrm{C})$, such as diarrhea (OR, 1.31; 95\% CI, $0.89-1.95$; $\mathrm{p}=0.17$ ) or 
nausea/vomiting (OR, 0.56; 95\% CI, 0.24-1.30; $\mathrm{p}=0.18$ ). There was no significant difference in change of electrocardiogram (OR, 1.16; 95\% CI, 0.94-1.42; $\mathrm{p}=0.16 ; \mathrm{I}^{2}=34 \%$ ) (Figure 4D), incidence of arrhythmia (OR, 1.28; 95\% CI, 0.94-1.74; $\mathrm{p}=0.12$ ), bradycardia/tachycardia (OR, 1.18; 95\% CI, 0.42-3.29; $\mathrm{p}=0.76$ ), or QT prolongation (OR, 1.06; 95\% CI, 0.80-1.40; $\mathrm{p}=0.69$ ) either between the two groups (patients treated with azithromycin and control).

A Respiratory support

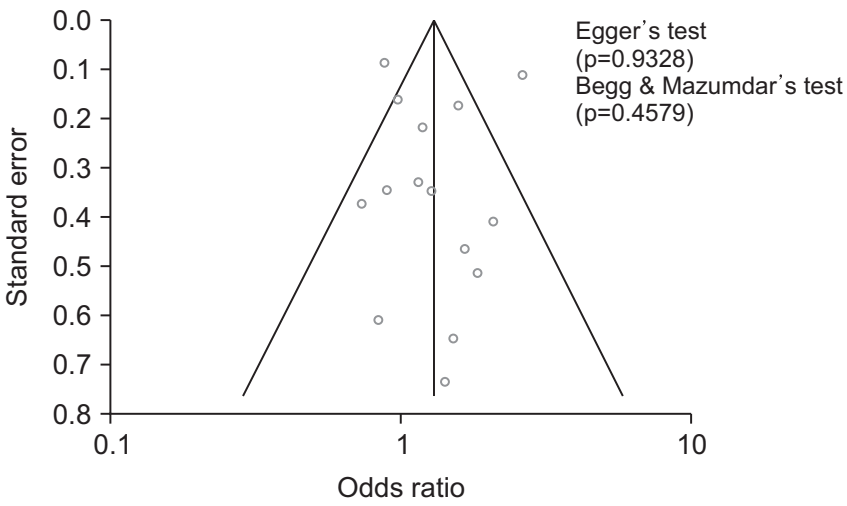

C Mortality rate

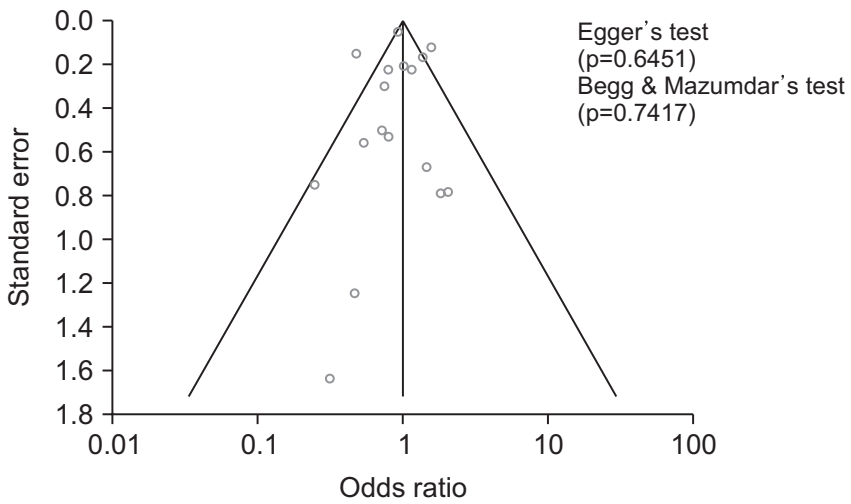

E Secondary infection

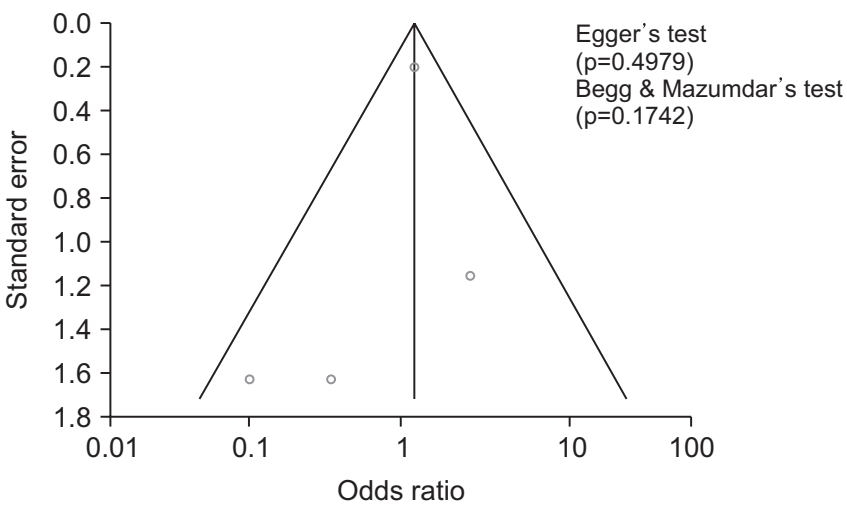

This meta-analysis found no evidence of publication bias (Figure 5) except for the assessment of gastrointestinal symptoms occurring in azithromycin-treated patients compared to those in the control. The rest of outcomes showed a symmetrical funnel plot which was further confirmed statistically ( $p>0.1$ ) by Begg and Mazumdar rank correlation test and Egger's test of the intercept. Sensitivity analysis was conducted with or without exclusion of a study that cause some concerns

B Hospitalization period

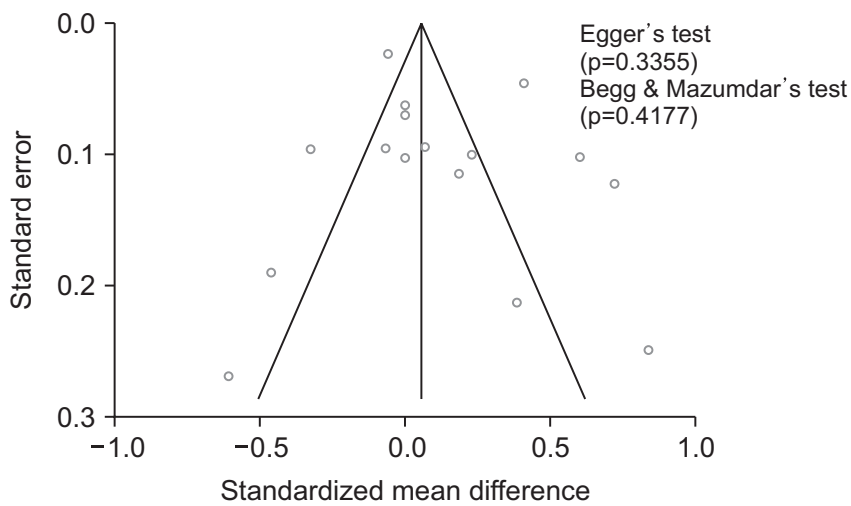

D ICU transfer

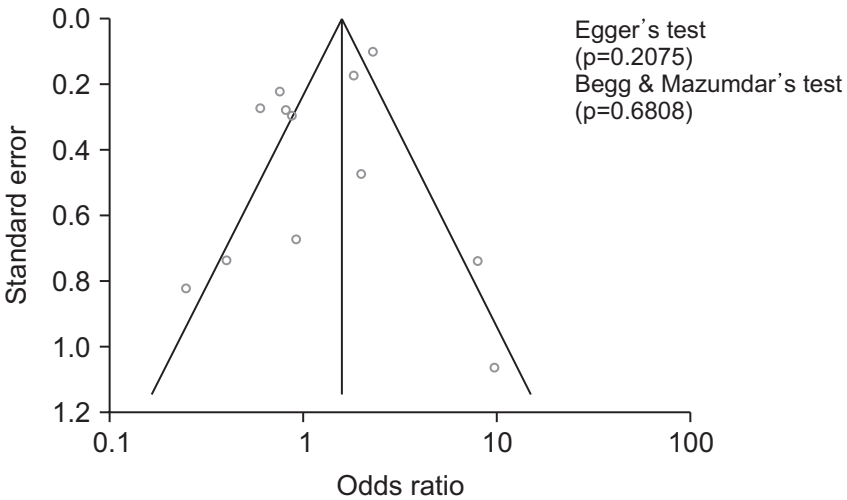

F Hypoglycemia

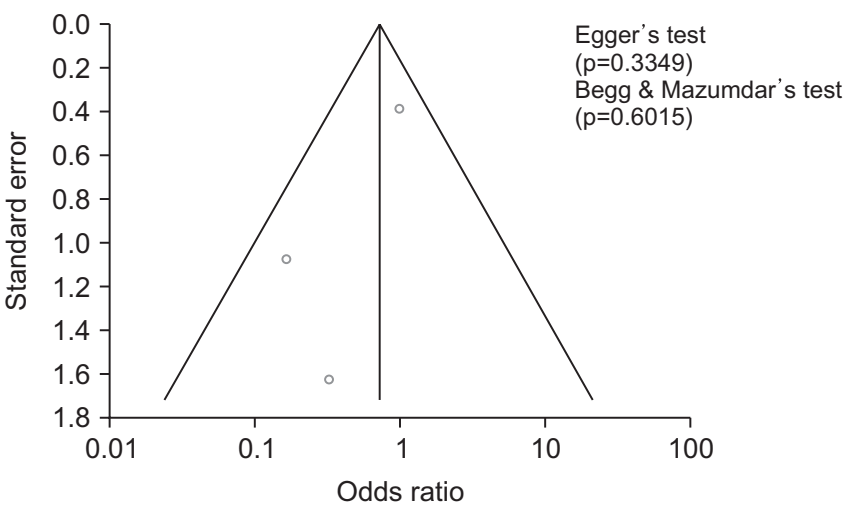

Figure 5. (A-H) Publication bias. Funnel plot presented the distribution of included studies. Asymmetrical plot indicated that publication bias was present. This was confirmed by Begg and Mazumdar rank correlation test and Egger's test of the intercept to determine the presence of publication bias statistically $(\mathrm{p}<0.1)$. ICU: intensive care unit. 
G Gastrointestinal symptoms

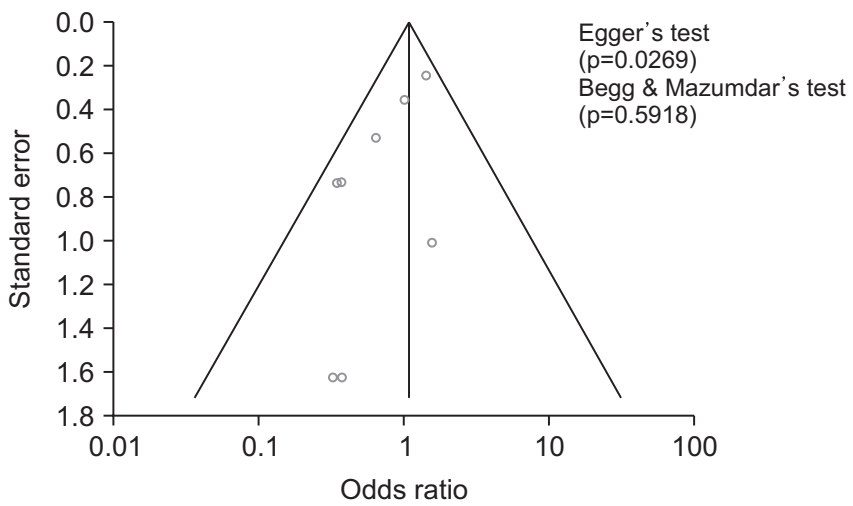

Figure 5. Continued.
H Changes in electrocardiogram

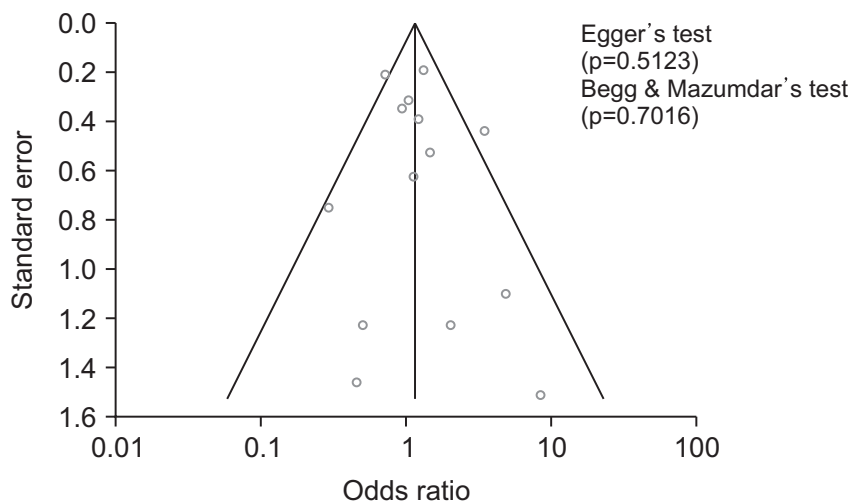

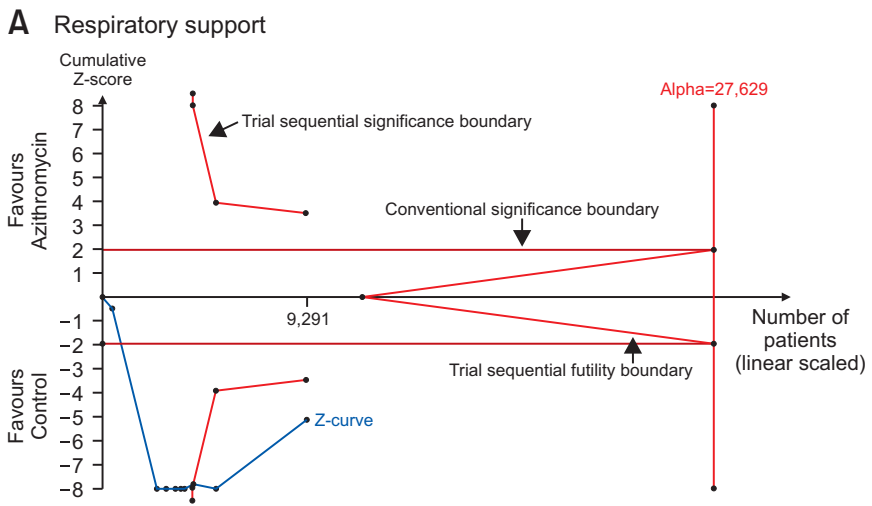

B Hospitalization period

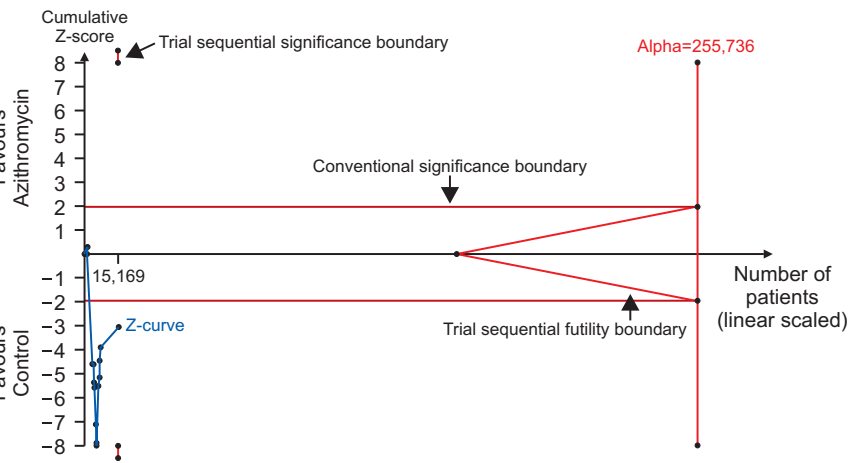

C Mortality rate
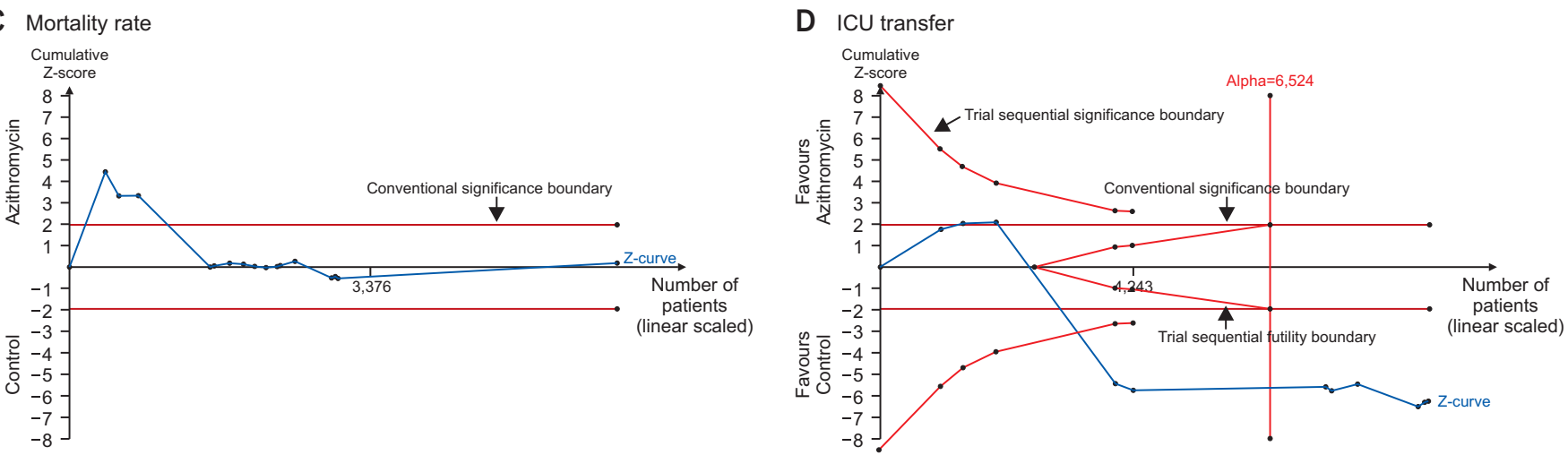

Figure 6. (A-H) Trial sequential analysis. Findings are represented by cumulative Z-curves. When Z-curves surpass the futility boundary, the level of evidence is adequate and further trials will be judged as futile. The level of evidence was judged to be adequate and conclusive if the Z-curves surpassed the conventional and trial sequential significance boundaries. On the contrary, when Z-curves did not cross any boundaries or only surpassed the conventional boundary, the level of evidence was inadequate and more trials would be needed to clarify the conclusion. The blue line represents the cumulative $\mathrm{Z}$-curve. The horizontal red line at $\mathrm{Z}=+1.96$ and $\mathrm{Z}=-1.96$ indicates the conventional meta-analysis boundary. The diagonal red line at the top and the bottom of the plot indicates the trial sequential significance boundary. The triangular red line on the right represents the trial sequential futility boundary. The vertical red line on the right indicates the required sample size for the meta-analysis.

of bias. Findings did not show any meaningful differences, indicating the stability of results from this meta-analysis.

TSA was performed to further investigate and confirm re- sults from this meta-analysis (Figure 6). All pooled analyses did not exceed the required sample size except in the assessment of ICU transfer. However, TSA confirmed that results 

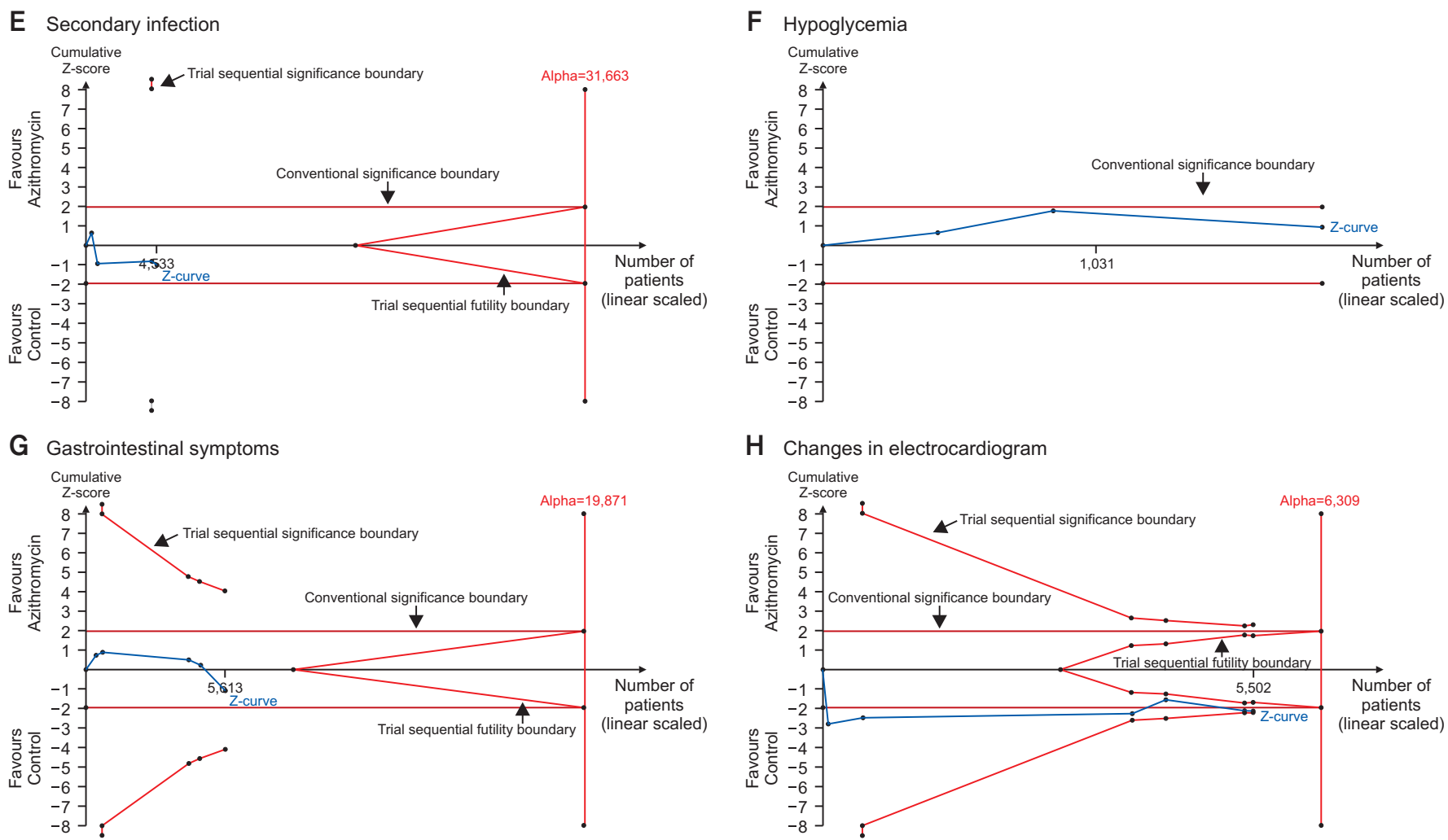

Figure 6. Continued.

of this meta-analysis evaluating the need of ICU transfer and respiratory support were conclusive as the cumulative Zcurve of outcomes surpassed both conventional and trial significance boundaries, indicating that type I and type II errors were avoided. On the contrary, pooled analysis evaluating the rest of outcomes was inconclusive as the cumulative Z-curve either surpassed the conventional boundary (but not the trial sequential significance boundary) or surpassed neither boundaries. Therefore, more clinical studies are needed to confirm these results.

\section{Confidence in cumulative evidence}

Studies included in this meta-analysis were randomized controlled trials (RCTs) and cohorts that indicated initial moderate-quality evidence in the GRADE system. The majority of RCTs were judged to have a low risk of bias according to RoB2 except in one study. Meanwhile, all included cohort studies were judged to have a good quality. Sensitivity analysis did not show any meaningful differences either when one study with some concerns of bias was omitted. Therefore, it could be concluded that results were unlikely to be affected by bias. No serious indirectness was found in this study that could affect study results. Publication bias was not present except in the meta-analysis evaluating gastrointestinal symp- toms that occurred after azithromycin treatment. There were substantial inconsistencies in results evaluating the efficacy of azithromycin due to high heterogeneity of studies caused by differences in the population. Although the CI of each outcome was unlikely to pose a problem, the majority of results from this meta-analysis caused some concerns regarding the precision of data as TSA was inconclusive. Overall, these included studies were had a low-to-very low quality of evidence. GRADE evidence profile is summarized in Table 3.

\section{Discussion}

COVID-19 patients who received azithromycin treatment were unlikely to have better outcomes than those who did not receive it. This meta-analysis demonstrated that azithromycin treatment was not significantly associated with a lower mortality, a shorter hospitalization period, a lower ICU transfer, or a less need for respiratory support. Azithromycin is a broadspectrum antibiotic widely used to treat lower respiratory tract infections ${ }^{3}$. The rationale for using azithromycin in COVID-19 treatment was probably due to its potential immunomodulatory, anti-inflammatory, and anti-viral properties ${ }^{3-5,34}$. It has been reported that patients with moderate-to-severe ARDS have significant clinical improvement after they are treated 


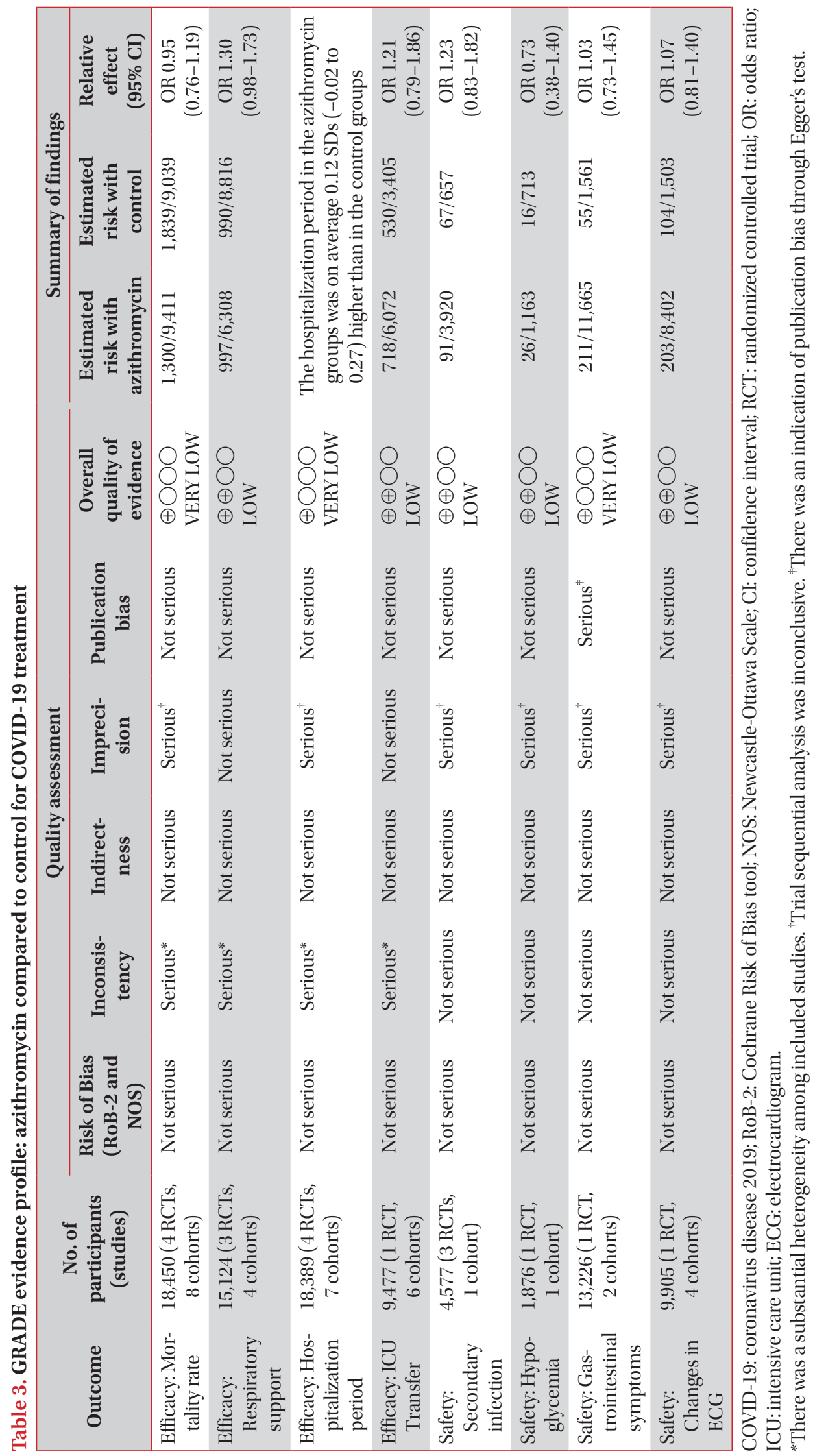


with azithromycin ${ }^{7}$. The widespread use of azithromycin in COVID-19 patients might be driven by the risk of bacterial superinfections in patients with a more severe disease ${ }^{9}$. However, this meta-analysis of subjects with mostly moderateto-severe COVID-19 showed no meaningful clinical benefits from azithromycin treatment. This might be due to a low rate of secondary infection among subjects included in this study or due to the fact that the effect of azithromycin was partially masked by the use of other antibiotics or standard COVID-19 treatment.

In terms of safety, azithromycin has a relatively safe profile. This meta-analysis suggested that the number of patients in the azithromycin group experiencing adverse events such as hypoglycemia, diarrhea, nausea/vomiting, arrhythmia, and secondary infections were similar to those in the control group. The risk of QT prolongation was not statistically significant either compared to previous studies showing a potential torsadogenic effect of azithromycin ${ }^{7,8}$.

The evidence generated from this study confirmed that azithromycin was not associated with a significant clinical improvement in COVID-19 patients. The lack of clinical benefits suggested that routine use of azithromycin should be ceased except in cases with evident bacterial pneumonia for which a combination of a beta-lactam and macrolide antibiotics is recommended ${ }^{35}$. However, it was unclear whether the quality of evidence from this meta-analysis was sufficient. Although overall pooled results were stable, effects were inconclusive for the majority of cases. Additional data are needed to confirm results of this study. There were substantial inconsistencies observed across studies, especially in the analysis evaluating the efficacy of azithromycin. It might be due to the heterogeneous nature of study subjects and the timing of outcome measurement. Despite some imprecision and heterogeneity in outcomes, this meta-analysis suggested a weak recommendation for using azithromycin as one treatment for COVID-19.

Azithromycin did not result in a superior clinical improvement for COVID-19 patients, although it was well-tolerated and safe to use. Due to a low quality of evidence presented in this meta-analysis, more clinical studies are needed to clearly elucidate the benefit of azithromycin for COVID-19 patients.

\section{Authors' Contributions}

Conceptualization: Glenardi, Mangkuliguna G, Natalia. Methodology: Glenardi, Mangkuliguna G, Natalia, Pramono LA. Formal analysis: Glenardi, Mangkuliguna G, Natalia, Pramono LA. Data curation: Glenardi, Mangkuliguna G, Natalia, Pramono LA. Software: Glenardi, Mangkuliguna G, Natalia. Validation: Glenardi, Mangkuliguna G, Natalia, Pramono LA. Investigation: Glenardi, Mangkuliguna G, Natalia, Pramono LA. Writing - original draft preparation: Glenardi, Mangkuliguna G, Natalia. Writing - review and editing: Glenardi, Mangku- liguna G, Natalia, Pramono LA. Approval of final manuscript: all authors.

\section{Conflicts of Interest}

No potential conflict of interest relevant to this article was reported.

\section{Funding}

No funding to declare.

\section{References}

1. WHO Coronavirus (COVID-19) Dashboard [Internet]. Geneva: World Health Organization; 2021 [cited 2021 Apr 16]. Available from: https://covid19.who.int/.

2. Bonam SR, Kaveri SV, Sakuntabhai A, Gilardin L, Bayry J. Adjunct immunotherapies for the management of severely ill COVID-19 patients. Cell Rep Med 2020;1:100016.

3. Zarogoulidis P, Papanas N, Kioumis I, Chatzaki E, Maltezos E, Zarogoulidis K. Macrolides: from in vitro anti-inflammatory and immunomodulatory properties to clinical practice in respiratory diseases. Eur J Clin Pharmacol 2012;68:479-503.

4. Min JY, Jang YJ. Macrolide therapy in respiratory viral infections. Mediators Inflamm 2012;2012:649570.

5. Lee N, Wong CK, Chan MC, Yeung ES, Tam WW, Tsang OT, et al. Anti-inflammatory effects of adjunctive macrolide treatment in adults hospitalized with influenza: a randomized controlled trial. Antiviral Res 2017;144:48-56.

6. Kawamura K, Ichikado K, Takaki M, Eguchi Y, Anan K, Suga M. Adjunctive therapy with azithromycin for moderate and severe acute respiratory distress syndrome: a retrospective, propensity score-matching analysis of prospectively collected data at a single center. Int J Antimicrob Agents 2018;51:91824.

7. Yang Z, Prinsen JK, Bersell KR, Shen W, Yermalitskaya L, Sidorova T, et al. Azithromycin causes a novel proarrhythmic syndrome. Circ Arrhythm Electrophysiol 2017;10:e003560.

8. Sultana J, Cutroneo PM, Crisafulli S, Puglisi G, Caramori G, Trifiro G. Azithromycin in COVID-19 patients: pharmacological mechanism, clinical evidence and prescribing guidelines. Drug Saf 2020;43:691-8.

9. Hall M, Pritchard M, Dankwa EA, Baillie JK, Carson G, Citarella BW, et al. ISARIC Clinical Data Report 20 November 2020. Preprint at: https://doi.org/10.1101/2020.07.17.201552182020 (2020).

10. Albani F, Fusina F, Giovannini A, Ferretti P, Granato A, Prezioso C, et al. Impact of azithromycin and/or hydroxychloroquine on hospital mortality in COVID-19. J Clin Med 2020;9: 
2800.

11. Arshad S, Kilgore P, Chaudhry ZS, Jacobsen G, Wang DD, Huitsing K, et al. Treatment with hydroxychloroquine, azithromycin, and combination in patients hospitalized with COVID-19. Int J Infect Dis 2020;97:396-403.

12. Bernardini A, Ciconte G, Negro G, Rondine R, Mecarocci V, Viva T, et al. Assessing QT interval in COVID-19 patients:safety of hydroxychloroquine-azithromycin combination regimen. Int J Cardiol 2021;324:242-8.

13. Cavalcanti AB, Zampieri FG, Rosa RG, Azevedo LCP, Veiga VC, Avezum A, et al. Hydroxychloroquine with or without azithromycin in mild-to-moderate Covid-19. N Engl J Med 2020;383:2041-52.

14. Furtado RHM, Berwanger O, Fonseca HA, Correa TD, Ferraz LR, Lapa MG, et al. Azithromycin in addition to standard of care versus standard of care alone in the treatment of patients admitted to the hospital with severe COVID-19 in Brazil (COALITION II): a randomised clinical trial. Lancet 2020;396:95967.

15. Lagier JC, Million M, Gautret P, Colson P, Cortaredona S, Giraud-Gatineau A, et al. Outcomes of 3,737 COVID-19 patients treated with hydroxychloroquine/azithromycin and other regimens in Marseille, France: a retrospective analysis. Travel Med Infect Dis 2020;36:101791.

16. Lauriola M, Pani A, Ippoliti G, Mortara A, Milighetti S, Mazen M, et al. Effect of combination therapy of hydroxychloroquine and azithromycin on mortality in patients with COVID-19. Clin Transl Sci 2020;13:1071-6.

17. Mercuro NJ, Yen CF, Shim DJ, Maher TR, McCoy CM, Zimetbaum PJ, et al. Risk of QT interval prolongation associated with use of hydroxychloroquine with or without concomitant azithromycin among hospitalized patients testing positive for coronavirus disease 2019 (COVID-19). JAMA Cardiol 2020;5: 1036-41.

18. Omrani AS, Pathan SA, Thomas SA, Harris TR, Coyle PV, Thomas CE, et al. Randomized double-blinded placebocontrolled trial of hydroxychloroquine with or without azithromycin for virologic cure of non-severe Covid-19. EClinicalMedicine 2020;29:100645.

19. Ozdemir IH, Ozlek B, Ozen MB, Gunduz R, Cetin N, Bilge AR. Hydroxychloroquine/azithromycin treatment, QT interval and ventricular arrhythmias in hospitalised patients with COVID-19. Int J Clin Pract 2021;75:e13896.

20. RECOVERY Collaborative Group. Azithromycin in patients admitted to hospital with COVID-19 (RECOVERY): a randomised, controlled, open-label, platform trial. Lancet 2021;397:605-12.

21. Rodriguez-Molinero A, Perez-Lopez C, Galvez-Barron C, Minarro A, Macho O, Lopez GF, et al. Observational study of azithromycin in hospitalized patients with COVID-19. PLoS One 2020;15:e238681.

22. Rosenberg ES, Dufort EM, Udo T, Wilberschied LA, Kumar J, Tesoriero J, et al. Association of treatment with hydroxychlo- roquine or azithromycin with in-hospital mortality in patients with COVID-19 in New York State. JAMA 2020;323:2493-502.

23. Saleh M, Gabriels J, Chang D, Soo Kim B, Mansoor A, Mahmood E, et al. Effect of chloroquine, hydroxychloroquine, and azithromycin on the corrected QT interval in patients with SARS-CoV-2 infection. Circ Arrhythm Electrophysiol 2020;13:e008662.

24. Sekhavati E, Jafari F, SeyedAlinaghi S, Jamalimoghadamsiahkali S, Sadr S, Tabarestani M, et al. Safety and effectiveness of azithromycin in patients with COVID-19: an open-label randomised trial. Int J Antimicrob Agents 2020;56:106143.

25. Seyhan AU, Doganay F, Yilmaz E, Topal NP, Ak R. Investigation of QT prolongation with hydroxychloroquine and azithromycin for the treatment of COVID-19. J Coll Physicians Surg Pak 2020;30:153-7.

26. Tanriverd IE, Cortuk M, Yildirim BZ, Ugur Chousein EG Turan D, Cinarka H, et al. Hydroxychloroquine plus azithromycin and early hospital admission are beneficial in COVID-19 patients: Turkish experience with real-life data. Turk J Med Sci 2021;51:10-5.

27. Echeverria-Esnal D, Martin-Ontiyuelo C, Navarrete-Rouco ME, De-Antonio Cusco M, Ferrandez O, Horcajada JP, et al. Azithromycin in the treatment of COVID-19: a review. Expert Rev Ant-Infect Ther 2021;19:147-63.

28. Moher D, Liberati A, Tetzlaff J, Altman DG; PRISMA Group. Preferred reporting items for systematic reviews and metaanalyses: the PRISMA statement. PLoS Med 2009;6:e1000097.

29. Sterne JA, Savovic J, Page MJ, Elbers RG, Blencowe NS, Boutron I, et al. RoB 2: a revised tool for assessing risk of bias in randomised trials. BMJ 2019;366:14898.

30. Wells GA, Shea B, O'Connell D, Peterson J, Welch V, Losos M, et al. The Newcastle-Ottawa Scale (NOS) for assessing the quality of nonrandomised studies in meta-analyses [Internet]. Ottawa: Ottawa Hospital; 2013 [cited 2021 Apr 16]. Available from: http://www.ohri.ca/programs/clinical_epidemiology/ oxford.asp.

31. Thorlund K, Engstrøm J, Wetterslev J, Brok J, Imberger G, Gluud C. User manual for trial sequential analysis (TSA) [Internet]. Copenhagen: Copenhagen Trial Unit, Centre for Clinical Intervention Research; 2011 [cited 2021 Apr 16]. Available from: http://www.ctu.dk/tsa.

32. Review Manager (RevMan), version 5.4. London: The Cochrane Collaboration; 2020.

33. MedCalc Software. MedCalc, version 19.5.1. Ostend: MedCalc Software Ltd.; 2020.

34. Rizk JG, Kalantar-Zadeh K, Mehra MR, Lavie CJ, Rizk Y, Forthal DN. Pharmaco-immunomodulatory therapy in COVID-19. Drugs 2020;80:1267-92.

35. Metlay JP, Waterer GW, Long AC, Anzueto A, Brozek J, Crothers K, et al. Diagnosis and treatment of adults with communityacquired pneumonia: an official clinical practice guideline of the American Thoracic Society and Infectious Diseases Society of America. Am J Respir Crit Care Med 2019;200:e45-67. 